\title{
NONCOMMUTATIVE BOYD INTERPOLATION THEOREMS
}

\author{
SJOERD DIRKSEN
}

\begin{abstract}
We present a new, elementary proof of Boyd's interpolation theorem. Our approach naturally yields a noncommutative version of this result and even allows for the interpolation of certain operators on $\ell^{1}$-valued noncommutative symmetric spaces. By duality we may interpolate several well-known noncommutative maximal inequalities. In particular we obtain a version of Doob's maximal inequality and the dual Doob inequality for noncommutative symmetric spaces. We apply our results to prove the BurkholderDavis-Gundy and Burkholder-Rosenthal inequalities for noncommutative martingales in these spaces.
\end{abstract}

\section{INTRODUCTION}

Symmetric Banach function spaces play a pivotal role in many fields of mathematical analysis, especially probability theory, interpolation theory and harmonic analysis. A cornerstone result in the interpolation theory of these spaces is the Boyd interpolation theorem, named after D.W. Boyd. Together with the CalderónMitjagin theorem, which characterizes the symmetric Banach function spaces which are an exact interpolation space for the couple $\left(L^{1}\left(\mathbb{R}_{+}\right), L^{\infty}\left(\mathbb{R}_{+}\right)\right)$, Boyd's theorem provides an invaluable tool for the analysis of symmetric spaces.

The history of Boyd's interpolation theorem begins with the announcement of Marcinkiewicz [28, shortly before his death, of an extension of the Riesz-Thorin theorem. Let us say that a sublinear operator $T$ is of Marcinkiewicz weak type $(p, p)$ if for any $f \in L^{p}\left(\mathbb{R}_{+}\right)$,

$$
d(v ; T f)^{\frac{1}{p}} \leq C v^{-1}\|f\|_{L^{p}\left(\mathbb{R}_{+}\right)} \quad(v>0),
$$

where $d(\cdot ; T f)$ denotes the distribution function of $T f$. Marcinkiewicz demonstrated that if a sublinear operator $T$ is simultaneously of Marcinkiewicz weak types $(p, p)$ and $(q, q)$ for $1 \leq p<q \leq \infty$, then $T$ is bounded on $L^{r}\left(\mathbb{R}_{+}\right)$, for any $p<r<q$. A full proof of this result was published years later by Zygmund [36, based on Marcinkiewicz' notes. Soon after, it was observed by Stein and Weiss [33] that Marcinkiewicz' result is valid for the larger class of operators which are simultaneously of weak types $(p, p)$ and $(q, q)$. Here $T$ is said to be of weak type $(p, p)$ if for any $f$ in the Lorentz space $L^{p, 1}\left(\mathbb{R}_{+}\right)$,

$$
d(v ; T f)^{\frac{1}{p}} \leq C v^{-1}\|f\|_{L^{p, 1}\left(\mathbb{R}_{+}\right)} .
$$

Received by the editors April 12, 2012 and, in revised form, March 12, 2013.

2010 Mathematics Subject Classification. Primary 46B70, 46L52, 46L53.

Key words and phrases. Boyd interpolation theorem, noncommutative symmetric spaces, $\Phi$-moment inequalities, Doob maximal inequality, Burkholder-Davis-Gundy inequalities, Burkholder-Rosenthal inequalities.

This research was supported by VICI subsidy 639.033.604 of the Netherlands Organisation for Scientific Research (NWO) and the Hausdorff Center for Mathematics. 
The class of sublinear operators which are simultaneously of weak types $(p, p)$ and $(q, q)$ was subsequently characterized by Calderón [6] as consisting of precisely those maps $T$ which satisfy

$$
\mu_{t}(T f) \leq C S_{p, q}(\mu(f))(t) \quad(t>0),
$$

where $\mu(f)$ denotes the decreasing rearrangement of $f$ and $S_{p, q}$ is a linear integral operator which is nowadays known as Calderón's operator. Finally, in [5] Boyd introduced two indices $p_{E}$ and $q_{E}$ for any symmetric Banach function space $E$ on $\mathbb{R}_{+}$ and showed that the operator $S_{p, q}$ is bounded on $E$ precisely when $p<p_{E} \leq q_{E}<q$. Together with Calderón's characterization, this yields Boyd's interpolation theorem: every sublinear operator which is simultaneously of weak types $(p, p)$ and $(q, q)$ is bounded on $E$ if and only if $p<p_{E} \leq q_{E}<q$.

In this paper we are concerned with obtaining a generalization of Boyd's result to noncommutative and, to a limited extent, also noncommutative vector-valued symmetric Banach function spaces. As it turns out, the original approach sketched above remains feasible in the noncommutative setting (see the appendix of this paper), but becomes problematic for noncommutative vector-valued spaces. We develop a new, elementary approach to Boyd's interpolation theorem for the class of Marcinkiewicz weak type operators. Our approach consists of two observations, which are close in spirit to the original approach. Firstly, we characterize the sublinear operators of simultaneous Marcinkiewicz weak types $(p, p)$ and $(q, q)$ as being exactly those which for some $\alpha>0$ satisfy the inequality

$$
d(\alpha v ; T f) \leq d\left(v ; \Theta_{p, q} f\right) \quad(v>0),
$$

where $\Theta_{p, q}$ is the linear operator defined by

$$
\Theta_{p, q} f(s, t)=f(s)\left(t^{-\frac{1}{q}} \chi_{(0,1)}(t)+t^{-\frac{1}{p}} \chi_{[1, \infty)}(t)\right) .
$$

Secondly, we show that $\Theta_{p, q}$ is bounded on $E$ if $p<p_{E} \leq q_{E}<q$. Our approach immediately extends to yield both a vector-valued and a noncommutative version of Boyd's result. Moreover, all results are valid for symmetric quasi-Banach function spaces. Thus we obtain Boyd's theorem and its extensions for the full scale of $L^{p}$-spaces.

Interestingly, our method even yields interpolation results for certain operators defined on noncommutative $\ell^{1}$ - and $\ell^{2}$-valued $L^{p}$-spaces in the sense of Pisier 31. In particular, it allows for the interpolation of noncommutative probabilistic inequalities such as the dual Doob inequality, in the noncommutative setting due to Junge [17, and the 'upper' noncommutative Khintchine inequalities, originally due to Lust-Piquard [25, 26]. In fact, our approach has its origins in the proof of the Khintchine inequalities for noncommutative symmetric spaces given in [7,8], which the author only later understood as Boyd-type interpolation results.

By adapting the duality argument in Junge's proof of the Doob maximal inequality for noncommutative $L^{p}$-spaces, we can dualize our noncommutative $\ell^{1}$-valued interpolation theorem to find an interpolation result for noncommutative maximal inequalities. In particular, we deduce a version of Doob's maximal inequality for a large class of noncommutative symmetric spaces. In the final section we utilize the latter inequality and its dual version to prove Burkholder-Davis-Gundy and Burkholder-Rosenthal inequalities, respectively, for noncommutative symmetric spaces. Our results extend the Burkholder-Gundy and Rosenthal inequalities 
established in [7], as well as the Burkholder-Rosenthal inequalities for noncommutative $L^{p}$-spaces and Lorentz spaces obtained in [18] and [15], respectively.

During the writing of this manuscript we discovered that an interpolation result for noncommutative $\Phi$-moment inequalities associated with Orlicz functions was proved recently in [1]. We discuss the connection of our work with this result and in fact show that many of our interpolation results have a ' $\Phi$-moment version'.

The paper is organized so that the first part, up to the classical Boyd interpolation theorem, can be read without any knowledge of noncommutative analysis.

\section{Symmetric quasi-Banach function spaces}

In this preliminary section we introduce symmetric quasi-Banach function spaces and discuss their most important properties. The results presented below are all well known for Banach function spaces, but not easy to find for quasi-Banach function spaces. We shall need the following well-known result due to T. Aoki and S. Rolewicz, which states that every quasi-normed vector space can be equipped with an equivalent $r$-norm (see e.g. 21] for a proof).

Theorem 2.1 (Aoki-Rolewicz). Let $X$ be a quasi-normed vector space. Then there is $a C>0$ and $0<r \leq 1$ such that for any $x_{1}, \ldots, x_{n} \in X$,

$$
\left\|\sum_{i=1}^{n} x_{i}\right\|_{X} \leq C\left(\sum_{i=1}^{n}\left\|x_{i}\right\|_{X}^{r}\right)^{\frac{1}{r}}
$$

Let $\tilde{S}\left(\mathbb{R}_{+}\right)$be the linear space of all measurable, a.e. finite functions $f$ on $\mathbb{R}_{+}$. For any $f \in \tilde{S}\left(\mathbb{R}_{+}\right)$we define its distribution function by

$$
d(v ; f)=\lambda\left(t \in \mathbb{R}_{+}:|f(t)|>v\right) \quad(v \geq 0),
$$

where $\lambda$ denotes Lebesgue measure. Let $S\left(\mathbb{R}_{+}\right)$be the subspace of all $f \in \tilde{S}\left(\mathbb{R}_{+}\right)$ such that $d(v ; f)<\infty$ for some $v>0$ and let $S_{0}\left(\mathbb{R}_{+}\right)$be the subspace of all $f \in S\left(\mathbb{R}_{+}\right)$with $d(v ; f)<\infty$ for all $v>0$. For $f \in S\left(\mathbb{R}_{+}\right)$we denote by $\mu(f)$ the decreasing rearrangement of $f$, defined by

$$
\mu_{t}(f)=\inf \{v>0: d(v ; f) \leq t\} \quad(t \geq 0) .
$$

For $f, g \in S\left(\mathbb{R}_{+}\right)$we say $f$ is submajorized by $g$, and write $f \prec \prec g$, if

$$
\int_{0}^{t} \mu_{s}(f) d s \leq \int_{0}^{t} \mu_{s}(g) d s, \quad \text { for all } t>0 .
$$

A (quasi-)normed linear subspace $E$ of $S\left(\mathbb{R}_{+}\right)$is called a (quasi-)Banach function space on $\mathbb{R}_{+}$if it is complete and if for $f \in S\left(\mathbb{R}_{+}\right)$and $g \in E$ with $|f| \leq|g|$ we have $f \in E$ and $\|f\|_{E} \leq\|g\|_{E}$. A (quasi-)Banach function space $E$ on $\mathbb{R}_{+}$is called symmetric if for $f \in S\left(\mathbb{R}_{+}\right)$and $g \in E$ with $\mu(f) \leq \mu(g)$ we have $f \in E$ and $\|f\|_{E} \leq\|g\|_{E}$. It is called fully symmetric if, in addition, for $f \in S\left(\mathbb{R}_{+}\right)$and $g \in E$ with $f \prec \prec g$ it follows that $f \in E$ and $\|f\|_{E} \leq\|g\|_{E}$.

A symmetric (quasi-)Banach function space is said to have a Fatou (quasi-)norm if for every net $\left(f_{\beta}\right)$ in $E$ and $f \in E$ satisfying $0 \leq f_{\beta} \uparrow f$ we have $\left\|f_{\beta}\right\|_{E} \uparrow\|f\|_{E}$. The space $E$ is said to have the Fatou property if for every net $\left(f_{\beta}\right)$ in $E$ satisfying $0 \leq f_{\beta} \uparrow$ and $\sup _{\beta}\left\|f_{\beta}\right\|_{E}<\infty$ the supremum $f=\sup _{\beta} f_{\beta}$ exists in $E$ and $\left\|f_{\beta}\right\|_{E} \uparrow$ $\|f\|_{E}$. We say that $E$ has order continuous (quasi-)norm if for every net $\left(f_{\beta}\right)$ in $E$ such that $f_{\beta} \downarrow 0$ we have $\left\|f_{\beta}\right\|_{E} \downarrow 0$. In the literature, a symmetric (quasi-)Banach 
function space is often called rearrangement invariant if it has order continuous (quasi-)norm or the Fatou property. We shall not use this terminology.

Let us finally discuss some results specific for symmetric Banach function spaces. The Köthe dual of a symmetric Banach function space $E$ is the Banach function space $E^{\times}$given by

$$
\begin{aligned}
& E^{\times}=\left\{g \in S\left(\mathbb{R}_{+}\right): \sup \left\{\int_{0}^{\infty}|f(t) g(t)| d t:\|f\|_{E} \leq 1\right\}<\infty\right\} ; \\
& \|g\|_{E^{\times}}=\sup \left\{\int_{0}^{\infty}|f(t) g(t)| d t:\|f\|_{E} \leq 1\right\}, \quad g \in E^{\times} .
\end{aligned}
$$

The space $E^{\times}$is fully symmetric and has the Fatou property. It is isometrically isomorphic to a closed subspace of $E^{*}$ via the map

$$
g \mapsto L_{g}, \quad L_{g}(f)=\int_{0}^{\infty} f(t) g(t) d t \quad(f \in E) .
$$

A symmetric Banach function space on $\mathbb{R}_{+}$has a Fatou norm if and only if $E$ embeds isometrically into its second Köthe dual $E^{\times \times}=\left(E^{\times}\right)^{\times}$. It has the Fatou property if and only if $E=E^{\times \times}$isometrically. It has order continuous norm if and only if it is separable, which is also equivalent to the statement $E^{*}=E^{\times}$. Moreover, a symmetric Banach function space which is separable or has the Fatou property is automatically fully symmetric. For proofs of these facts and more details we refer to $4,23,24$.

2.1. Boyd indices. We now discuss the Boyd indices, which were introduced by D.W. Boyd in [5]. For any $0<a<\infty$ we define the dilation operator $D_{a}$ on $S\left(\mathbb{R}_{+}\right)$ by

$$
\left(D_{a} f\right)(s)=f(a s) \quad\left(s \in \mathbb{R}_{+}\right) .
$$

The following lemma is well known for symmetric Banach function spaces (cf. 23]).

Lemma 2.2. Let $E$ be a symmetric quasi-Banach function space on $\mathbb{R}_{+}$. Then, for every $0<a<\infty, D_{a}$ defines a bounded linear operator on $E$. Moreover, $a \mapsto\left\|D_{a}\right\|$ is a decreasing, submultiplicative function on $\mathbb{R}_{+}$.

Proof. Since $\mu(f)$ is decreasing, we have for any $a \leq b$,

$$
D_{b} \mu(f)(s)=\mu_{b s}(f) \leq \mu_{a s}(f)=D_{a} \mu(f)(s) .
$$

Hence, if $D_{a}$ is bounded on $E$, then $D_{b}$ is bounded on $E$ as well and $\left\|D_{b}\right\| \leq\left\|D_{a}\right\|$. In particular, $\left\|D_{a}\right\|$ is bounded on $E$ if $a \geq 1$ and $\left\|D_{a}\right\| \leq 1$. Moreover, it suffices to show that $D_{1}$ is bounded on $E$ for every $n \in \mathbb{N}$.

Fix $n \in \mathbb{N}$, let $f \in E_{+}$and let $f_{i}, 1 \leq i \leq n$, be mutually disjoint functions having the same distribution function as $f$. Then $D_{\frac{1}{n}} f$ and $\sum_{i=1}^{n} f_{i}$ have the same distribution function. Indeed,

$$
\begin{aligned}
\lambda\left(t \in \mathbb{R}_{+}:\left(D_{\frac{1}{n}} f\right)(t)>v\right) & =n \lambda\left(t \in \mathbb{R}_{+}: f(t)>v\right) \\
& =\sum_{i=1}^{n} \lambda\left(t \in \mathbb{R}_{+}: f_{i}(t)>v\right) \\
& =\lambda\left(t \in \mathbb{R}_{+}: \sum_{i=1}^{n} f_{i}(t)>v\right) .
\end{aligned}
$$


Since $E$ is symmetric it follows that $D_{\frac{1}{n}} f \in E$. Moreover, by Theorem 2.1, there exists some $c>0$ and $0<p \leq 1$ such that

$$
\left\|D_{\frac{1}{n}} f\right\|_{E}=\left\|\sum_{i=1}^{n} f_{i}\right\|_{E} \leq c\left(\sum_{i=1}^{n}\left\|f_{i}\right\|_{E}^{p}\right)^{\frac{1}{p}}=c n^{\frac{1}{p}}\|f\|_{E} .
$$

From the above it is clear that $a \mapsto\left\|D_{a}\right\|$ is decreasing and, since $D_{a b}=D_{a} D_{b}$ if $a \leq b$, submultiplicative.

Define the lower Boyd index $p_{E}$ of $E$ by

$$
p_{E}=\lim _{s \rightarrow \infty} \frac{\log s}{\log \left\|D_{\frac{1}{s}}\right\|}
$$

and the upper Boyd index $q_{E}$ of $E$ by

$$
q_{E}=\lim _{s \downarrow 0} \frac{\log s}{\log \left\|D_{\frac{1}{s}}\right\|} .
$$

By the Aoki-Rolewicz theorem $E$ admits an equivalent $p$-norm for some $0<p \leq 1$, and, as observed in (3), for every $n \geq 1$ we have

$$
\left\|D_{\frac{1}{n}} f\right\|_{E} \leq n^{\frac{1}{p}}\|f\|_{E}
$$

and therefore $p \leq p_{E}$. In particular we have $0<p_{E} \leq q_{E} \leq \infty$, and if $E$ is a symmetric Banach function space, then $1 \leq p_{E} \leq q_{E} \leq \infty$. One may show that the Boyd indices can alternatively be expressed as

$$
p_{E}=\sup \left\{p>0: \exists c>0 \forall 0<a \leq 1\left\|D_{a} f\right\|_{E} \leq c a^{-\frac{1}{p}}\|f\|_{E}\right\}
$$

and

$$
q_{E}=\inf \left\{q>0: \exists c>0 \forall a \geq 1\left\|D_{a} f\right\|_{E} \leq c a^{-\frac{1}{q}}\|f\|_{E}\right\} .
$$

We shall need the following duality for Boyd indices (see [23, Theorem II.4.11). If $E$ is a symmetric Banach function space with Fatou norm, then

$$
\frac{1}{p_{E}}+\frac{1}{q_{E \times}}=1, \quad \frac{1}{p_{E^{\times}}}+\frac{1}{q_{E}}=1 .
$$

2.2. Convexity and concavity. Let $0<p, q \leq \infty$. A symmetric quasi-Banach function space $E$ is said to be $p$-convex if there exists a constant $C>0$ such that for any finite sequence $\left(f_{i}\right)_{i=1}^{n}$ in $E$ we have

$$
\left\|\left(\sum_{i=1}^{n}\left|f_{i}\right|^{p}\right)^{\frac{1}{p}}\right\|_{E} \leq C\left(\sum_{i=1}^{n}\left\|f_{i}\right\|_{E}^{p}\right)^{\frac{1}{p}} \quad(\text { if } 0<p<\infty)
$$

or

$$
\left\|\max _{1 \leq i \leq n}\left|f_{i}\right|\right\|_{E} \leq C \max _{1 \leq i \leq n}\left\|f_{i}\right\|_{E} \quad(\text { if } p=\infty) .
$$

The least constant $M^{(p)}$ for which this inequality holds is called the p-convexity constant of $E$.

A symmetric quasi-Banach function space $E$ is said to be $q$-concave if there exists a constant $C>0$ such that for any finite sequence $\left(f_{i}\right)_{i=1}^{n}$ in $E$ we have

$$
\left(\sum_{i=1}^{n}\left\|f_{i}\right\|_{E}^{q}\right)^{\frac{1}{q}} \leq C\left\|\left(\sum_{i=1}^{n}\left|f_{i}\right|^{q}\right)^{\frac{1}{q}}\right\|_{E} \quad(\text { if } 0<q<\infty)
$$


or

$$
\max _{1 \leq i \leq n}\left\|f_{i}\right\|_{E} \leq C\left\|\max _{1 \leq i \leq n}\left|f_{i}\right|\right\|_{E} \quad(\text { if } q=\infty) .
$$

The least constant $M_{(q)}$ for which this inequality holds is called the q-concavity constant of $E$. It is clear that every quasi-Banach function space is $\infty$-concave with $M_{(\infty)}=1$ and any Banach function space is 1-convex with $M^{(1)}=1$.

For $1 \leq r<\infty$, let the $r$-concavification and $r$-convexification of $E$ be defined by

$$
\begin{aligned}
& E_{(r)}:=\left\{g \in S\left(\mathbb{R}_{+}\right):|g|^{\frac{1}{r}} \in E\right\},\|g\|_{E_{(r)}}=\left\||g|^{\frac{1}{r}}\right\|_{E}^{r}, \\
& E^{(r)}:=\left\{g \in S\left(\mathbb{R}_{+}\right):|g|^{r} \in E\right\},\|g\|_{E^{(r)}}=\left\||g|^{r}\right\|_{E}^{\frac{1}{r}}
\end{aligned}
$$

respectively. As is shown in 24] (p. 53), if $E$ is a Banach function space, then $E^{(r)}$ is a Banach function space. In general, $E_{(r)}$ is only a quasi-Banach function space. Using that $\mu\left(|f|^{s}\right)=\mu(f)^{s}$ for any $f \in S\left(\mathbb{R}_{+}\right)$and $0<s<\infty$, one sees that $E^{(r)}$ and $E_{(r)}$ are symmetric if $E$ is symmetric. From the definitions one easily shows that if $E$ is $p$-convex and $q$-concave for $0<p \leq q \leq \infty$, then $E^{(r)}$ is $p r$-convex and $q r$-concave and $E_{(r)}$ is $\frac{p}{r}$-convex and $\frac{q}{r}$-concave. It is also clear from the definitions that

$$
p_{E_{(r)}}=\frac{1}{r} p_{E}, q_{E_{(r)}}=\frac{1}{r} q_{E}, p_{E^{(r)}}=r p_{E}, q_{E^{(r)}}=r q_{E} .
$$

We conclude this section by discussing two concrete classes of symmetric quasiBanach function spaces in more detail.

Example 2.1 (Lorentz spaces $L^{p, q}$ ). Let $0<p, q \leq \infty$. The Lorentz space $L^{p, q}$ is the subspace of all $f$ in $S\left(\mathbb{R}_{+}\right)$such that

$$
\|f\|_{L^{p, q}}=\left\{\begin{array}{cl}
\left(\int_{0}^{\infty} t^{\frac{q}{p}-1} \mu_{t}(f)^{q} d t\right)^{\frac{1}{q}} & (0<q<\infty), \\
\sup _{0<t<\infty} t^{\frac{1}{p}} \mu_{t}(f) & (q=\infty)
\end{array}\right.
$$

is finite. If $1 \leq q \leq p<\infty$ or $p=q=\infty$, then $L^{p, q}$ is a fully symmetric Banach function space. If $1<p<\infty$ and $p \leq q \leq \infty$, then $L^{p, q}$ can be equivalently renormed to become a fully symmetric Banach function space (4, Theorem 4.6). However, in general $L^{p, q}$ is only a symmetric quasi-Banach function space [20]. By the monotone convergence theorem, $L^{p, q}$ has the Fatou property. Its Boyd indices are determined by the first exponent, $p_{L^{p, q}}=q_{L^{p, q}}=p$. The Lorentz space $L^{p, p}$ coincides with the Lebesgue space $L^{p}$. The spaces $L^{p, \infty}$ are referred to as weak $L^{p}$-spaces.

Example 2.2 (Orlicz spaces). Let $\Phi:[0, \infty) \rightarrow[0, \infty]$ be a Young's function, i.e., a convex, continuous and increasing function satisfying $\Phi(0)=0$ and $\lim _{t \rightarrow \infty} \Phi(t)=$ $\infty$. The Orlicz space $L_{\Phi}$ is the subspace of all $f$ in $S\left(\mathbb{R}_{+}\right)$such that for some $k>0$,

$$
\int_{0}^{\infty} \Phi\left(\frac{|f(t)|}{k}\right) d t<\infty
$$

If we equip $L_{\Phi}$ with the Luxemburg norm

$$
\|f\|_{L_{\Phi}}=\inf \left\{k>0: \int_{0}^{\infty} \Phi\left(\frac{|f(t)|}{k}\right) d t \leq 1\right\}
$$


then $L_{\Phi}$ is a symmetric Banach function space with the Fatou property [4,24]. The Boyd indices of $L_{\Phi}$ can be computed in terms of $\Phi$. Indeed, let

$$
M_{\Phi}(t)=\sup _{s>0} \frac{\Phi(t s)}{\Phi(s)}
$$

and define the Matuszewska-Orlicz indices by

$$
p_{\Phi}=\lim _{t \downarrow 0} \frac{\log M_{\Phi}(t)}{\log t}, \quad q_{\Phi}=\lim _{t \rightarrow \infty} \frac{\log M_{\Phi}(t)}{\log t} .
$$

One can show that $p_{\Phi}=p_{L_{\Phi}}$ and $q_{\Phi}=q_{L_{\Phi}}$; see e.g. the proof of [27], Theorem 4.2. For our discussion of $\Phi$-moment inequalities we will need the following results on Orlicz functions. We say that an Orlicz function satisfies the global $\Delta_{2}$-condition if for some constant $C>0$,

$$
\Phi(2 t) \leq C \Phi(t) \quad(t \geq 0) .
$$

Under this condition we have, for any $\alpha \geq 0$,

$$
\Phi(\alpha t) \lesssim_{\alpha, \Phi} \Phi(t) \quad(t \geq 0) .
$$

One can show ([27], Theorem 3.2(b)) that (66) is equivalent to the assumption $q_{\Phi}<\infty$, which in turn holds if and only if

$$
\sup _{t>0} \frac{t \Phi^{\prime}(t)}{\Phi(t)}<\infty .
$$

Finally, we shall use the following characterization of Boyd's indices for Orlicz spaces $([27$, Theorem 6.4$)$ :

$$
\begin{aligned}
& p_{\Phi}=\sup \left\{p>0: \int_{0}^{t} s^{-p} \Phi(s) \frac{d s}{s} \lesssim_{p, \Phi} t^{-p} \Phi(t) \forall t>0\right\}, \\
& q_{\Phi}=\inf \left\{q>0: \int_{t}^{\infty} s^{-q} \Phi(s) \frac{d s}{s} \lesssim_{q, \Phi} t^{-q} \Phi(t) \forall t>0\right\} .
\end{aligned}
$$

We refer to [4, 23, 24, for many more concrete examples of symmetric quasiBanach function spaces.

\section{Characterization of Marcinkiewicz Weak type operators}

In this section we establish a key observation which essentially reduces the proof of Boyd's theorem and its noncommutative extensions to proving a certain inequality for distribution functions, which is stated in Lemma 3.7 below. This observation moreover leads to a characterization of the subconvex operators which are simultaneously of weak types $(p, p)$ and $(q, q)$; see Theorem 3.8

For $0<p, q \leq \infty$ we define the functions $\phi_{q}, \psi_{p}, \theta_{p, q}: \mathbb{R}_{+} \rightarrow \mathbb{R}_{+}$by

$$
\begin{aligned}
\phi_{q}(t) & =t^{-\frac{1}{q}} \chi_{(0,1)}(t) & & (t>0) ; \\
\psi_{p}(t) & =t^{-\frac{1}{p}} \chi_{[1, \infty)}(t) & & (t>0) \\
\theta_{p, q}(t) & =\psi_{p}(t)+\phi_{q}(t) & & (t>0) .
\end{aligned}
$$

Here it is understood that $\phi_{\infty}=\chi_{(0,1)}$. Corresponding to these functions we define three linear operators $\Phi_{q}, \Psi_{p}, \Theta_{p, q}: S\left(\mathbb{R}_{+}\right) \rightarrow \tilde{S}\left(\mathbb{R}_{+} \times \mathbb{R}_{+}\right)$by

$$
\Phi_{q}(f)=f \otimes \phi_{q}, \quad \Psi_{p}(f)=f \otimes \psi_{p}, \quad \Theta_{p, q}(f)=f \otimes \theta_{p, q} .
$$

The following observation is a reformulation of [7], Lemma 4.3. 
Lemma 3.1. Let $E$ be a symmetric quasi-Banach function space on $\mathbb{R}_{+}$and let $0<q<\infty$. If $q_{E}<q$, then $\Phi_{q}$ is bounded from $E\left(\mathbb{R}_{+}\right)$into $E\left(\mathbb{R}_{+} \times \mathbb{R}_{+}\right)$. Conversely, if $\Phi_{q}$ is bounded, then $q_{E} \leq q$.

Clearly $\Phi_{\infty}$ is an isometry from $E\left(\mathbb{R}_{+}\right)$into $E\left(\mathbb{R}_{+} \times \mathbb{R}_{+}\right)$for every symmetric quasi-Banach function space $E$.

The corresponding result for the lower Boyd index reads as follows. In the proof and later on, we use $\chi_{A}$ to denote the indicator of a set $A$.

Lemma 3.2. Let $E$ be a symmetric quasi-Banach function space on $\mathbb{R}_{+}$and let $0<p<\infty$. If $p<p_{E}$, then $\Psi_{p}$ is bounded from $E\left(\mathbb{R}_{+}\right)$into $E\left(\mathbb{R}_{+} \times \mathbb{R}_{+}\right)$. Conversely, if $\Psi_{p}$ is bounded, then $p \leq p_{E}$.

Proof. Fix $p<p_{0}<p_{E}$. It clearly suffices to prove

$$
\left\|f \otimes \psi_{p}\right\|_{E\left(\mathbb{R}_{+} \times \mathbb{R}_{+}\right)} \leq c_{p, E}\|f\|_{E\left(\mathbb{R}_{+}\right)},
$$

for any $f \in E_{+}$. Observe that $f \otimes \chi_{\left[2^{n}, 2^{n+1}\right)}$ has the same distribution on $\mathbb{R}_{+} \times \mathbb{R}_{+}$ as $D_{2^{-n}} f$ on $\mathbb{R}_{+}$. Hence,

$$
\begin{aligned}
\left\|f \otimes \psi_{p}\right\|_{E\left(\mathbb{R}_{+} \times \mathbb{R}_{+}\right)} & \leq\left\|f(s) \sum_{n=0}^{\infty} 2^{-\frac{n}{p}} \chi_{\left[2^{n}, 2^{n+1}\right)}(t)\right\|_{E\left(\mathbb{R}_{+} \times \mathbb{R}_{+}\right)} \\
& \leq C\left(\sum_{n=0}^{\infty} 2^{-\frac{n r}{p}}\left\|f(s) \chi_{\left[2^{n}, 2^{n+1}\right)}(t)\right\|_{E\left(\mathbb{R}_{+} \times \mathbb{R}_{+}\right)}^{r}\right)^{\frac{1}{r}} \\
& =C\left(\sum_{n=0}^{\infty} 2^{-\frac{n r}{p}}\left\|D_{2^{-n}} f\right\|_{E\left(\mathbb{R}_{+}\right)}^{r}\right)^{\frac{1}{r}},
\end{aligned}
$$

where $C$ and $0<r \leq 1$ are as in (11). By (4), there is some constant $C_{p_{0}}>0$ such that

$$
\left\|D_{u}\right\| \leq C_{p_{0}} u^{-\frac{1}{p_{0}}} \quad(0<u \leq 1) .
$$

Hence,

$$
\begin{aligned}
\left\|f \otimes \psi_{p}\right\|_{E\left(\mathbb{R}_{+} \times \mathbb{R}_{+}\right)} & \leq \quad c C_{p_{0}}\left(\sum_{n=0}^{\infty} 2^{-\frac{n r}{p}} 2^{\frac{n r}{p_{0}}}\|f\|_{E\left(\mathbb{R}_{+}\right)}^{r}\right)^{\frac{1}{r}} \\
& \lesssim_{p, E}\|f\|_{E\left(\mathbb{R}_{+}\right)}
\end{aligned}
$$

as $\frac{1}{p_{0}}-\frac{1}{p}<0$.

For the second assertion, notice first that $\mu\left(D_{s}(f)\right)=D_{s} \mu(f)$ for all $0<s<\infty$ and $f \in E$. Therefore, it suffices to show that there is a constant $c>0$ such that for all $0<s \leq 1$ and $f \in E_{+}$we have $\left\|D_{s} f\right\|_{E} \leq c s^{-\frac{1}{p}}\|f\|_{E}$. If $1 \leq a<\infty$, then

$$
\begin{aligned}
\left\|f \otimes \psi_{p}\right\|_{E\left(\mathbb{R}_{+} \times \mathbb{R}_{+}\right)} & \geq\left\|f(s) t^{-\frac{1}{p}} \chi_{[a, 2 a)}(t)\right\|_{E\left(\mathbb{R}_{+} \times \mathbb{R}_{+}\right)} \\
& \geq\left\|f(s)(2 a)^{-\frac{1}{p}} \chi_{[a, 2 a)}(t)\right\|_{E\left(\mathbb{R}_{+} \times \mathbb{R}_{+}\right)} \\
& =2^{-\frac{1}{p}} a^{-\frac{1}{p}}\left\|D_{a^{-1}} f\right\|_{E\left(\mathbb{R}_{+}\right)},
\end{aligned}
$$

where we use that $f \otimes \chi_{[a, 2 a)}$ has the same distribution on $\mathbb{R}_{+} \times \mathbb{R}_{+}$as $D_{a^{-1}} f$ on $\mathbb{R}_{+}$. By (9) we arrive at

$$
\left\|D_{a^{-1}} f\right\|_{E} \leq 2^{\frac{1}{p}} a^{\frac{1}{p}}\left\|f \otimes \psi_{p}\right\|_{E\left(\mathbb{R}_{+} \times \mathbb{R}_{+}\right)}{ }_{p, E} a^{\frac{1}{p}}\|f\|_{E} .
$$

Since this holds for any $1 \leq a<\infty$, we conclude that $p \leq p_{E}$. 
As a result of Lemmas 3.1 and 3.2 we find the following novel expressions for Boyd's indices:

$$
\begin{aligned}
& p_{E}=\sup \left\{p>0: \exists C>0 \forall f \in E\left\|\Psi_{p}(f)\right\|_{E\left(\mathbb{R}_{+} \times \mathbb{R}_{+}\right)} \leq C\|f\|_{E\left(\mathbb{R}_{+}\right)}\right\} \\
& q_{E}=\inf \left\{q>0: \exists C>0 \forall f \in E\left\|\Phi_{q}(f)\right\|_{E\left(\mathbb{R}_{+} \times \mathbb{R}_{+}\right)} \leq C\|f\|_{E\left(\mathbb{R}_{+}\right)}\right\} .
\end{aligned}
$$

Moreover, we have the following result.

Corollary 3.3. Let $0<p<q<\infty$ and let $E$ be a symmetric quasi-Banach function space on $\mathbb{R}_{+}$. If $p<p_{E} \leq q_{E}<q$, then $\Theta_{p, q}$ is bounded from $E\left(\mathbb{R}_{+}\right)$into $E\left(\mathbb{R}_{+} \times \mathbb{R}_{+}\right)$. Conversely, if $\Theta_{p, q}$ is bounded, then $p \leq p_{E} \leq q_{E} \leq q$.

The bound for the operator norm of $\Theta_{p, q}$ given in the proof of Corollary 3.3 can be improved in specific situations. For example, if $0<p<r<q \leq \infty$, then one easily calculates that

$$
\left\|\Theta_{p, q}\right\|_{L^{r} \rightarrow L^{r}}=\left(\frac{p}{r-p}+\frac{q}{q-r}\right)^{\frac{1}{r}}
$$

We now compute the distribution function of $\Phi_{q}(f), \Psi_{p}(f)$ and $\Theta_{p, q}(f)$. The first was already done in [7, Lemma 4.4.

Lemma 3.4. Let $0<q<\infty$. If $f \in S\left(\mathbb{R}_{+}\right)_{+}$, then for every $v>0$,

$$
d\left(v ; \Phi_{q}(f)\right)=\int_{\{f \leq v\}}\left(\frac{f(s)}{v}\right)^{q} d s+d(v ; f)
$$

and

$$
d\left(v ; \Phi_{\infty}(f)\right)=d(v ; f) .
$$

Lemma 3.5. Let $0<p<\infty$ and $f \in S\left(\mathbb{R}_{+}\right)_{+}$. If $d(v ; f)<\infty$, then

$$
d\left(v ; \Psi_{p}(f)\right)=\int_{\{f>v\}}\left(\frac{f(s)}{v}\right)^{p} d s-d(v ; f) .
$$

Proof. Using a change of variable,

$$
\begin{aligned}
\lambda((s, t) \in & \left.\mathbb{R}_{+} \times \mathbb{R}_{+}: f(s) \psi_{p}(t)>v\right) \\
& =\int_{1}^{\infty} \lambda\left(s \in \mathbb{R}_{+}: f(s) t^{-\frac{1}{p}}>v\right) d t \\
& =\int_{1}^{\infty} \lambda\left(s \in \mathbb{R}_{+}: \frac{f(s)}{v}>t^{\frac{1}{p}}\right) d t \\
& =\int_{1}^{\infty} \lambda\left(s \in \mathbb{R}_{+}: \frac{f(s)}{v}>u\right) p u^{p-1} d u \\
& =\left\|\frac{f}{v}\right\|_{L^{p}\left(\mathbb{R}_{+}\right)}^{p}-\int_{0}^{1} \lambda\left(s \in \mathbb{R}_{+}: \frac{f(s)}{v}>u\right) p u^{p-1} d u
\end{aligned}
$$


Observe that

$$
\begin{aligned}
\int_{0}^{1} \lambda(s & \left.\in \mathbb{R}_{+}: \frac{f(s)}{v}>u\right) p u^{p-1} d u \\
& =\int_{0}^{\infty} \lambda\left(s \in \mathbb{R}_{+}: \min \left\{\frac{f(s)}{v}, 1\right\}>u\right) p u^{p-1} d u \\
& =\left\|\min \left\{v^{-1} f, 1\right\}\right\|_{L^{p}\left(\mathbb{R}_{+}\right)}^{p}=\int_{\{f \leq v\}}\left(\frac{f(s)}{v}\right)^{p} d s+d(v ; f),
\end{aligned}
$$

which gives the conclusion.

Corollary 3.6. Let $0<p, q<\infty$. If $f \in S\left(\mathbb{R}_{+}\right)_{+}$, then for any $v>0$,

$$
d\left(v ; \Theta_{p, q}(f)\right)=\int_{\{f>v\}}\left(\frac{f(s)}{v}\right)^{p} d s+\int_{\{f \leq v\}}\left(\frac{f(s)}{v}\right)^{q} d s
$$

and

$$
d\left(v ; \Theta_{p, \infty}(f)\right)=\int_{\{f>v\}}\left(\frac{f(s)}{v}\right)^{p} d s .
$$

Proof. Since $f \otimes \phi_{q}$ and $f \otimes \psi_{p}$ have disjoint supports we have $d\left(v ; \Phi_{q}(f)\right)+$ $d\left(v ; \Psi_{p}(f)\right)=d\left(v ; \Theta_{p, q}(f)\right)$. Therefore, if $d(v ; f)<\infty$, then (10) and (11) follow immediately from Lemmas 3.4 and 3.5. On the other hand, for any $v>0$,

$$
d(v ; f) \leq d\left(v ; \Phi_{q}(f)\right) \leq d\left(v ; \Theta_{p, q}(f)\right)
$$

and

$$
d(v ; f) \leq \int_{\{f>v\}}\left(\frac{f(s)}{v}\right)^{p} d s .
$$

Hence, if $d(v ; f)=\infty$, then both sides of (10) and (11) are equal to $\infty$.

Lemma 3.7. Let $E$ be a symmetric quasi-Banach function space on $\mathbb{R}_{+}$. Let $\alpha>0$ and $f \in E_{+}$. Suppose that either $p<p_{E} \leq q_{E}<q<\infty$ or $p<p_{E}$ and $q=\infty$ and $g \in S\left(\mathbb{R}_{+}\right)$satisfies

$$
d(\alpha v ; g) \leq d\left(v ; \Theta_{p, q} f\right) \quad(v>0)
$$

Then $g \in E$ and

$$
\|g\|_{E} \leq \alpha\left\|\Theta_{p, q}\right\|_{E \rightarrow E}\|f\|_{E}
$$

Proof. We take right continuous inverses in (12) to obtain

$$
\mu_{t}(g) \leq \alpha \mu_{t}\left(\Theta_{p, q} f\right) \quad(t \geq 0) .
$$

As $E$ is symmetric, it follows from Corollary 3.3 that $g \in E$ and, moreover,

$$
\|g\|_{E} \leq \alpha\left\|\Theta_{p, q} f\right\|_{E\left(\mathbb{R}_{+} \times \mathbb{R}_{+}\right)} \leq \alpha\left\|\Theta_{p, q}\right\|_{E \rightarrow E}\|f\|_{E} .
$$

The following result is reminiscent of Calderón's characterization of weak type operators (see Theorem A.1 for a noncommutative extension). Recall that if $D$ is a convex set in $S\left(\mathbb{R}_{+}\right)$, then an operator $T: D \rightarrow S\left(\mathbb{R}_{+}\right)$is called subconvex if for any $f, g \in D$ and $t \in[0,1]$ we have

$$
T(t f+(1-t) g) \leq t T(f)+(1-t) T(g) .
$$


Theorem 3.8. Let $0<p \leq q \leq \infty$. A subconvex operator $T: L^{p}\left(\mathbb{R}_{+}\right)_{+}+$ $L^{q}\left(\mathbb{R}_{+}\right)_{+} \rightarrow S\left(\mathbb{R}_{+}\right)$is simultaneously of Marcinkiewicz weak types $(p, p)$ and $(q, q)$, i.e.,

$$
\|T f\|_{L^{r, \infty}\left(\mathbb{R}_{+}\right)} \leq C_{r}\|f\|_{L^{r}\left(\mathbb{R}_{+}\right)} \quad\left(f \in L^{r}\left(\mathbb{R}_{+}\right)_{+}, r=p, q\right)
$$

if and only if there is some $\alpha>0$ such that for all $f \in S\left(\mathbb{R}_{+}\right)$,

$$
d(\alpha v ; T f) \leq d\left(v ; \Theta_{p, q}(f)\right) \quad(v>0) .
$$

Proof. Suppose that (13) holds and fix $v>0$. We may assume that $d\left(v ; \Theta_{p, q}(f)\right)<$ $\infty$, for otherwise there is nothing to prove. By Corollary 3.6 it follows that $f \chi_{\{f>v\}} \in L^{p}\left(\mathbb{R}_{+}\right)$and $f \chi_{\{f \leq v\}} \in L^{q}\left(\mathbb{R}_{+}\right)$. If $C_{p, q}=\max \left\{C_{p}, C_{q}\right\}$, then by subconvexity,

$$
\begin{aligned}
d\left(2 C_{p, q} v ; T f\right) & \leq d\left(2 C_{p, q} v ; \frac{1}{2} T\left(2 f \chi_{\{f \leq v\}}\right)+\frac{1}{2} T\left(2 f \chi_{\{f>v\}}\right)\right) \\
& \leq d\left(2 C_{p, q} v ; T\left(2 f \chi_{\{f \leq v\}}\right)\right)+d\left(2 C_{p, q} v ; T\left(2 f \chi_{\{f>v\}}\right)\right) .
\end{aligned}
$$

By (13) and Corollary 3.6.

$$
\begin{aligned}
& d\left(2 C_{p, q} v ; T f\right) \\
& \quad \leq\left(2 C_{p, q} v\right)^{-q} C_{q}^{q}\left\|2 f \chi_{\{f \leq v\}}\right\|_{L^{q}\left(\mathbb{R}_{+}\right)}^{q}+\left(2 C_{p, q} v\right)^{-p} C_{p}^{p}\left\|2 f \chi_{\{f>v\}}\right\|_{L^{p}\left(\mathbb{R}_{+}\right)}^{p} \\
& \quad \leq d\left(v ; \Theta_{p, q} f\right) .
\end{aligned}
$$

Suppose now that (14) holds. If $q<\infty$, then by Corollary 3.6.

$$
d(\alpha v ; T f) \leq d\left(v ; \Theta_{p, q}(f)\right)=\int_{\{f \leq v\}} v^{-q} f(s)^{q} d s+\int_{\{f>v\}} v^{-p} f(s)^{p} d s .
$$

Since $p \leq q$ we have

$$
\left(v^{-1} f\right)^{p} \chi_{\{f>v\}} \leq\left(v^{-1} f\right)^{q} \chi_{\{f>v\}}, \quad\left(v^{-1} f\right)^{q} \chi_{\{f \leq v\}} \leq\left(v^{-1} f\right)^{p} \chi_{\{f \leq v\}},
$$

and therefore

$$
d(\alpha v ; T f) \leq v^{-r}\|f\|_{L^{r}\left(\mathbb{R}_{+}\right)}^{r} \quad(r=p, q) .
$$

On the other hand, if $q=\infty$, then it is clear that

$$
d(\alpha v ; T f) \leq d\left(v ; \Theta_{p, \infty}(f)\right)=\int_{\{f>v\}} v^{-p} f(s)^{p} d s \leq v^{-p}\|f\|_{L^{p}\left(\mathbb{R}_{+}\right)}^{p} .
$$

Moreover, for any $v>0$ we have

$$
d\left(\alpha v ; T\left(f \chi_{\{f \leq v\}}\right)\right)=0 .
$$

Applying this for $v=\|f\|_{\infty}$ yields

$$
T f \leq \alpha\|f\|_{\infty} \quad \text { a.e. }
$$

This completes the proof.

The following result shows that inequality (12) also implies $\Phi$-moment inequalities.

Lemma 3.9. Let $\Phi$ be an Orlicz function on $\mathbb{R}_{+}$which satisfies the global $\Delta_{2}$ condition. Let $\alpha>0$ and $f \in\left(L_{\Phi}\right)_{+}$. Suppose that either $p<p_{\Phi} \leq q_{\Phi}<q<\infty$ or $p<p_{\Phi}$ and $q=\infty$ and $g \in S\left(\mathbb{R}_{+}\right)$satisfies (12). Then $g \in L_{\Phi}$ and

$$
\int_{0}^{\infty} \Phi(|g(t)|) d t \lesssim_{p, q, \Phi} \int_{0}^{\infty} \Phi(f(t)) d t .
$$


Proof. Suppose that $q_{\Phi}<q<\infty$. Let $\lambda_{f}$ denote the pull-back measure on $\mathbb{R}_{+}$ associated with $f$ and $\lambda$. By Corollary 3.6 we can rewrite (12) as

$$
d(\alpha v ; g) \leq v^{-q} \int_{0}^{v} t^{q} d \lambda_{f}(t)+v^{-p} \int_{v}^{\infty} t^{p} d \lambda_{f}(t) .
$$

Integrating with respect to $\Phi$ and using Fubini's theorem yield

$$
\begin{aligned}
\int_{0}^{\infty} & \Phi(|g(t)|) d t \\
& \lesssim_{\Phi} \int_{0}^{\infty} v^{-q} \int_{0}^{v} t^{q} d \lambda_{f}(t) d \Phi(v)+\int_{0}^{\infty} v^{-p} \int_{v}^{\infty} t^{p} d \lambda_{f}(t) d \Phi(v) \\
& =\int_{0}^{\infty} \int_{t}^{\infty} v^{-q} t^{q} d \Phi(v) d \lambda_{f}(t)+\int_{0}^{\infty} \int_{0}^{t} v^{-p} t^{p} d \Phi(v) d \lambda_{f}(t) .
\end{aligned}
$$

By (7) and (8), we find

$$
\int_{t}^{\infty} v^{-q} d \Phi(v) \lesssim_{\Phi} \int_{t}^{\infty} v^{-q} \Phi(v) \frac{d v}{v} \lesssim_{q, \Phi} t^{-q} \Phi(t) .
$$

Similarly,

$$
\int_{0}^{t} v^{-p} d \Phi(v) \lesssim_{p, \Phi} t^{-p} \Phi(t) .
$$

We conclude that

$$
\int_{0}^{\infty} \Phi(|g(t)|) d t \lesssim_{p, q, \Phi} \int_{0}^{\infty} \Phi(t) d \lambda_{f}(t)=\int_{0}^{\infty} \Phi(f(t)) d t .
$$

The statement for $q=\infty$ is proved analogously.

Remark 3.10. From the presented proof it is clear that the result in Lemma 3.9, and hence the $\Phi$-moment inequalities discussed below, remain valid if $\Phi$ is nonconvex, provided that it satisfies (6) and (7), and $p_{\Phi}, q_{\Phi}$ are understood as in (8). It should be noted that in this case $L_{\Phi}$ is in general no longer a quasi-Banach space.

Boyd's interpolation theorem for Marcinkiewicz weak type operators, as well as a $\Phi$-moment version, are now an immediate consequence of the previous observations.

Theorem 3.11. Fix $0<p \leq q \leq \infty$. Let $T: L^{p}\left(\mathbb{R}_{+}\right)_{+}+L^{q}\left(\mathbb{R}_{+}\right)_{+} \rightarrow S\left(\mathbb{R}_{+}\right)$ be a subconvex operator of Marcinkiewicz weak types $(p, p)$ and $(q, q)$. If $E$ is a symmetric quasi-Banach function space on $\mathbb{R}_{+}$and either $p<p_{E} \leq q_{E}<q<\infty$ or $p<p_{E}$ and $q=\infty$ holds, then

$$
\|T f\|_{E\left(\mathbb{R}_{+}\right)} \leq 2\left\|\Theta_{p, q}\right\|_{E \rightarrow E} \max \left\{C_{p}, C_{q}\right\}\|f\|_{E\left(\mathbb{R}_{+}\right)} \quad\left(f \in E\left(\mathbb{R}_{+}\right)_{+}\right) .
$$

On the other hand, if $\Phi$ is an Orlicz function on $\mathbb{R}_{+}$satisfying the global $\Delta_{2}$ condition and either $p<p_{\Phi} \leq q_{\Phi}<q<\infty$ or $p<p_{\Phi}$ and $q=\infty$ holds, then

$$
\int_{0}^{\infty} \Phi(|T f(t)|) d t \lesssim_{\Phi, C_{p}, C_{q}} \int_{0}^{\infty} \Phi(f(t)) d t \quad\left(f \in\left(L_{\Phi}\right)_{+}\right) .
$$

Proof. As we have seen in the proof of Theorem 3.8 .

$$
d\left(2 \max \left\{C_{p}, C_{q}\right\} v ; T f\right) \leq d\left(v ; \Theta_{p, q} f\right) .
$$

The assertions now follow from Lemmas 3.7 and 3.9. respectively. 
Remark 3.12. Theorem 3.11 can be extended to a vector-valued interpolation theorem using the following simple trick contained in the proof of [3], Lemma 1. Suppose that $X, Y$ are Banach spaces and $T: L^{p, 1}\left(\mathbb{R}_{+} ; X\right)+L^{q, 1}\left(\mathbb{R}_{+} ; X\right) \rightarrow S\left(\mathbb{R}_{+} ; Y\right)$ satisfies

$$
\|T f\|_{L^{r, \infty}\left(\mathbb{R}_{+} ; Y\right)} \leq C_{r}\|f\|_{L^{r, 1}\left(\mathbb{R}_{+} ; X\right)} \quad\left(f \in L^{r, 1}\left(\mathbb{R}_{+} ; X\right), r=p, q\right) .
$$

For a fixed $f$ set $k(f)=\left(f /\|f\|_{X}\right) \chi_{\{f \neq 0\}}$ and define the subconvex operator

$$
S g=\|T(g k(f))\|_{Y} \quad\left(g \in L^{p, 1}\left(\mathbb{R}_{+}\right)+L^{q, 1}\left(\mathbb{R}_{+}\right)\right) .
$$

Since $\|k(f)\|_{X}=1$, it follows that

$$
\|S g\|_{L^{r, \infty}\left(\mathbb{R}_{+}\right)} \leq C_{r}\|g k(f)\|_{L^{r, 1}\left(\mathbb{R}_{+} ; X\right)}=C_{r}\|g\|_{L^{r, 1}\left(\mathbb{R}_{+}\right)} \quad\left(g \in L^{r, 1}\left(\mathbb{R}_{+}\right), r=p, q\right),
$$

and hence by the scalar-valued Boyd interpolation theorem,

$$
\|S g\|_{E} \leq 2 \max \left\{C_{p}, C_{q}\right\}\left\|\Theta_{p, q}\right\|\|g\|_{E} \quad(g \in E) .
$$

Taking $g=\|f\|_{X}$ yields

$$
\|T f\|_{E\left(\mathbb{R}_{+} ; Y\right)} \leq 2 \max \left\{C_{p}, C_{q}\right\}\left\|\Theta_{p, q}\right\|\|f\|_{E\left(\mathbb{R}_{+} ; X\right)} \quad\left(f \in E\left(\mathbb{R}_{+} ; X\right)\right) .
$$

\section{Noncommutative Boyd interpolation theOREMS}

In this section we prove a noncommutative version of Boyd's theorem, Theorem 4.8 below. We first recall some terminology and preliminary results for noncommutative symmetric spaces. Let $\mathcal{M}$ be a semi-finite von Neumann algebra acting on a complex Hilbert space $H$, which is equipped with a normal, semi-finite, faithful trace $\tau$. The distribution function of a closed, densely defined operator $x$ on $H$, which is affiliated with $\mathcal{M}$, is given by

$$
d(v ; x)=\tau\left(e^{|x|}(v, \infty)\right) \quad(v \geq 0),
$$

where $e^{|x|}$ is the spectral measure of $|x|$. The decreasing rearrangement of $x$ is defined by

$$
\mu_{t}(x)=\inf \{v>0: d(v ; x) \leq t\} \quad(t \geq 0) .
$$

We say that $x$ is $\tau$-measurable if $d(v ; x)<\infty$ for some $v>0$. We let $S(\tau)$ be the linear space of all $\tau$-measurable operators, which is a metrizable, complete topological *-algebra with respect to the measure topology. We denote by $S_{0}(\tau)$ the linear subspace of all $x \in S(\tau)$ such that $d(v ; x)<\infty$ for all $v>0$. One can introduce a partial order on the linear subspace $S(\tau)_{h}$ of all self-adjoint operators in $S(\tau)$ by setting, for a self-adjoint operator $x$,

$$
x \geq 0 \text { if and only if }\langle x \xi, \xi\rangle_{H} \geq 0 \text { for all } \xi \in D(x),
$$

where $D(x)$ is the domain of $x$ in $H$. We write $x \leq y$ for $x, y \in S(\tau)_{h}$ if and only if $y-x \geq 0$. Under this partial ordering $S(\tau)_{h}$ is a partially ordered vector space. Let $S(\tau)_{+}$denote the positive cone of all $x \in S(\tau)_{h}$ satisfying $x \geq 0$. It can be shown that $S(\tau)_{+}$is closed with respect to the measure topology ([11), Proposition 1.4).

Throughout our exposition, we will tacitly use many properties of distribution functions and decreasing rearrangements. For the convenience of the reader we collect these facts in the following two propositions. The first result is essentially contained in the proof of [30], Theorem 1. 
Proposition 4.1. If $x, y \in S(\tau)$, then:

(a) $d(v ; x)=d(v ; \mu(x))$ for all $v \geq 0$;

(b) $d(v+w ; x+y) \leq d(v ; x)+d(w ; y)$ for all $v, w \geq 0$;

(c) if $|x| \leq|y|$, then $d(v ; x) \leq d(v ; y)$ for all $v \geq 0$.

The following properties of decreasing rearrangements can be found in [13. If $p$ is a projection in $\mathcal{M}$, then we let $p^{\perp}:=\mathbf{1}-p$ denote its orthogonal complement.

Proposition 4.2. If $x, y \in S(\tau)$, then:

(a) $\mu_{t}(\lambda x)=|\lambda| \mu_{t}(x)$ for all $\lambda \in \mathbb{C}$ and $t \geq 0$;

(b) $\mu_{s+t}(x+y) \leq \mu_{s}(x)+\mu_{t}(y)$ for all $s, t \geq 0$;

(c) if $|x| \leq|y|$, then $\mu_{t}(x) \leq \mu_{t}(y)$ for all $t \geq 0$;

(d) $\mu_{t}(u x v) \leq\|u\| \mu_{t}(x)\|y\|$ for all $u, v \in \mathcal{M}$ and $t \geq 0$.

If $e=e^{|x|}(v, \infty)$, then

(e) $\mu_{t}(|x| e)=\mu_{t}(x) \chi_{[0, \tau(e))}(t)$ for all $t \geq 0$;

(f) $\mu_{t}\left(|x| e^{\perp}\right)=\mu_{t+\tau(e)}(x)$ for all $t \geq 0$, provided $\tau(e)<\infty$.

Finally, suppose that $\phi:[0, \infty) \rightarrow[0, \infty)$ is an increasing function which is leftcontinuous on $(0, \infty)$ and satisfies $\phi(0)=0$. If we define $\phi(\infty):=\lim _{t \rightarrow \infty} \phi(t)$, then

(g) $\mu(\phi(|x|))=\phi(\mu(x))$ on $[0, \infty)$.

For a symmetric (quasi-)Banach function space $E$ on $\mathbb{R}_{+}$, we define

$$
E(\mathcal{M}, \tau):=\left\{x \in S(\tau):\|\mu(x)\|_{E}<\infty\right\} .
$$

We usually denote $E(\mathcal{M}, \tau)$ by $E(\mathcal{M})$ for brevity. We call $E(\mathcal{M})$ the noncommutative (quasi-)Banach function space associated with $E$ and $\mathcal{M}$. In the quasi-Banach case these spaces were first considered by $\mathrm{Xu}$ in [35]. The following fundamental result is proved in [22, Theorem 8.11 (see also [11,35] for earlier proofs of this result under additional assumptions).

Theorem 4.3. If $E$ is a symmetric (quasi-)Banach function space on $\mathbb{R}_{+}$which is $p$-convex for some $0<p<\infty$, then $E(\mathcal{M})$ defines a $p$-convex (quasi-)Banach space under the (quasi-)norm $\|x\|_{E(\mathcal{M})}:=\|\mu(x)\|_{E}$. The space $E(\mathcal{M})$ is continuously embedded in $S(\tau)$ with respect to the measure topology.

Using the construction above, we obtain noncommutative versions of many important spaces in analysis, such as $L^{p}$-spaces, weak $L^{p}$-spaces, Lorentz spaces and Orlicz spaces. For more details on measurable operators we refer to [12, 13, 30, and for the theory of noncommutative symmetric spaces to 912,22 .

We will now proceed to prove the noncommutative version of Boyd's theorem. We first show that the noncommutative symmetric space $E(\mathcal{M})$ is intermediate for the couple $\left(L^{p}(\mathcal{M}), L^{q}(\mathcal{M})\right)$ if $p<p_{E} \leq q_{E}<q$, using the following observation.

Lemma 4.4. Let $0<p<q \leq \infty$ and let $E$ be a symmetric quasi-Banach function space $\mathbb{R}_{+}$which is $r$-convex for some $0<r<\infty$. If $E(\mathcal{M}) \subset L^{p}(\mathcal{M})+L^{q}(\mathcal{M})$, then

$$
\|x\|_{L^{p}(\mathcal{M})+L^{q}(\mathcal{M})} \lesssim_{p, q, E}\|x\|_{E(\mathcal{M})} \quad(x \in E(\mathcal{M})) .
$$

Proof. By Theorem 2.1, there exists an equivalent $s$-norm on $E(\mathcal{M})$ for some $0<$ $s \leq 1$. Suppose the assertion is not true. Then there exist $x_{n} \in E(\mathcal{M})_{+}$such that $\left\|x_{n}\right\|_{E(\mathcal{M})} \leq 1$, but $\left\|x_{n}\right\|_{L^{p}(\mathcal{M})+L^{q}(\mathcal{M})}>n^{2 / s+1}$ for all $n \geq 1$. By completeness 
it follows that $\sum_{n>1} n^{-2 / s} x_{n}$ converges in $E(\mathcal{M})$ to some $x \in E(\mathcal{M})_{+}$, and since $E(\mathcal{M}) \subset L^{p}(\mathcal{M})+L^{q}(\mathcal{M})$ we have $x \in\left(L^{p}(\mathcal{M})+L^{q}(\mathcal{M})\right)+$. But $n^{-2 / s} x_{n} \leq x$ and so $n<n^{-2 / s}\left\|x_{n}\right\|_{L^{p}(\mathcal{M})+L^{q}(\mathcal{M})} \leq\|x\|_{L^{p}(\mathcal{M})+L^{q}(\mathcal{M})}$, a contradiction.

Lemma 4.5. Let $0<p<q \leq \infty$ and let $E$ be a symmetric quasi-Banach function space $\mathbb{R}_{+}$which is $r$-convex for some $0<r<\infty$. If $0<p<p_{E}$ and either $q_{E}<q<\infty$ or $q=\infty$, then

$$
L^{p}(\mathcal{M}) \cap L^{q}(\mathcal{M}) \subset E(\mathcal{M}) \subset L^{p}(\mathcal{M})+L^{q}(\mathcal{M}),
$$

with continuous inclusions.

Proof. If $x \in E(\mathcal{M})$, then by Corollary 3.3 we have $\Theta_{p, q} \mu(x) \in E$ and hence $d\left(v ; \Theta_{p, q} \mu(x)\right)<\infty$ for some $v>0$. If $e_{v}=e^{|x|}[0, v]$, then by Proposition 4.2

$$
\left\|x e_{v}\right\|_{L^{q}(\mathcal{M})}^{q}=\int_{\{\mu(x) \leq v\}} \mu_{t}(x)^{q} d t, \quad\left\|x e_{v}^{\perp}\right\|_{L^{p}(\mathcal{M})}^{p}=\int_{\{\mu(x)>v\}} \mu_{t}(x)^{p} d t .
$$

It therefore follows from Corollary 3.6 that

$$
v^{-q}\left\|x e_{v}\right\|_{L^{q}(\mathcal{M})}^{q}+v^{-p}\left\|x e_{v}^{\perp}\right\|_{L^{p}(\mathcal{M})}^{p}=d\left(v ; \Theta_{p, q} \mu(x)\right)<\infty .
$$

Hence $x \in L^{p}(\mathcal{M})+L^{q}(\mathcal{M})$. By Lemma 4.4 this implies that $E(\mathcal{M}) \subset L^{p}(\mathcal{M})+$ $L^{q}(\mathcal{M})$ continuously.

Suppose now that $q=\infty$. Pick $v>0$ such that $d\left(v ; \Theta_{p, \infty} \mu(x)\right)<\infty$. Then $x e_{v} \in \mathcal{M}$ and $x e_{v}^{\perp} \in L^{p}(\mathcal{M})$ since by Proposition 4.2 and Corollary 3.6.

$$
v^{-p}\left\|x e_{v}^{\perp}\right\|_{L^{p}(\mathcal{M})}^{p}=v^{-p} \int_{\{\mu(x)>v\}} \mu_{t}(x)^{p} d t=d\left(v ; \Theta_{p, \infty} \mu(x)\right) .
$$

By Lemma 4.4 we conclude that $E(\mathcal{M})$ embeds continuously into $L^{p}(\mathcal{M})+\mathcal{M}$.

The first inclusion is immediate from the commutative case (see e.g. 24, Proposition 2.b.3), as $\left(L^{p} \cap L^{q}\right)(\mathcal{M})=L^{p}(\mathcal{M}) \cap L^{q}(\mathcal{M})$.

To formulate our main result the following definition is convenient. The notion of subconvexity given below weakens the notion of sublinear operators on spaces of measurable operators introduced by Q. Xu (see 14], where it first appeared in published form).

Definition 4.6. Let $\mathcal{M}$ and $\mathcal{N}$ be von Neumann algebras equipped with normal, semi-finite, faithful traces $\tau$ and $\sigma$, respectively. Let $D$ be a convex subset of $S(\tau)$. A map $T: D \rightarrow S(\sigma)_{h}$ is called midpoint convex if

$$
T\left(\frac{1}{2} x+\frac{1}{2} y\right) \leq \frac{1}{2} T(x)+\frac{1}{2} T(y)
$$

for all $x, y \in D$. A map $U: D \rightarrow S(\sigma)$ is called midpoint subconvex if for every $x, y \in D$ there exist partial isometries $u, v \in \mathcal{N}$ such that

$$
\left|U\left(\frac{1}{2} x+\frac{1}{2} y\right)\right| \leq \frac{1}{2} u^{*}|U x| u+\frac{1}{2} v^{*}|U y| v .
$$

It is a well-known fact (see e.g. [13], Lemma 4.3) that for any $x, y \in S(\sigma)$ there are partial isometries $u, v \in \mathcal{N}$ such that

$$
|x+y| \leq u^{*}|x| u+v^{*}|y| v .
$$

Therefore, any linear map is (midpoint) subconvex. 
For further reference we state Chebyshev's inequality.

Lemma 4.7 (Chebyshev's inequality). Let $0<q<\infty$. If $x \in L^{q}(\mathcal{M})$, then

$$
d(v ; x) \leq v^{-q}\|x\|_{L^{q}(\mathcal{M})}^{q} \quad(v>0) .
$$

For any $0<r<\infty$,

$$
\|x\|_{L^{r, \infty}(\mathcal{M})}=\sup _{t>0} t^{\frac{1}{r}} \mu_{t}(x)=\sup _{v>0} v d(v ; x)^{\frac{1}{r}},
$$

so Chebyshev's inequality implies that $L^{r}(\mathcal{M}) \subset L^{r, \infty}(\mathcal{M})$ contractively.

Theorem 4.8. Let $E$ be a symmetric quasi-Banach function space on $\mathbb{R}_{+}$which is $s$-convex for some $0<s<\infty$. Let $\mathcal{M}, \mathcal{N}$ be von Neumann algebras equipped with normal, semi-finite, faithful traces $\tau$ and $\sigma$, respectively. Suppose that $0<p<q \leq$ $\infty$ and let $T: L^{p}(\mathcal{M})_{+}+L^{q}(\mathcal{M})_{+} \rightarrow S(\sigma)$ be a midpoint subconvex map such that for some constants $C_{p}, C_{q}>0$ depending only on $p$ and $q$, respectively,

$$
\|T x\|_{L^{r, \infty}(\mathcal{N})} \leq C_{r}\|x\|_{L^{r}(\mathcal{M})} \quad\left(x \in L^{r}(\mathcal{M})_{+}, r=p, q\right) .
$$

If $p<p_{E} \leq q_{E}<q<\infty$ or $p<p_{E}$ and $q=\infty$, then

$$
\|T x\|_{E(\mathcal{N})} \leq 2\left\|\Theta_{p, q}\right\| \max \left\{C_{p}, C_{q}\right\}\|x\|_{E(\mathcal{M})} \quad\left(x \in E(\mathcal{M})_{+}\right) .
$$

The same result holds if $T: L^{p}(\mathcal{M})_{+}+L^{q}(\mathcal{M})_{+} \rightarrow S(\sigma)_{h}$ is a midpoint convex map satisfying (17).

Remark 4.9. Property (17) is clearly the noncommutative version of Marcinkiewicz weak type $(r, r)$. The reader should be warned, however, that in the noncommutative literature it is nowadays customary to simply refer to this property as 'weak type $(r, r)^{\prime}$.

Proof. We may assume that $\max \left\{C_{p}, C_{q}\right\} \leq 1$. By Lemma $4.5 T$ is well defined on $E(\mathcal{M})_{+}$. Let $x \in E(\mathcal{M})_{+}$and let $e_{v}=e^{x}[0, v]$. By midpoint subconvexity, there exist partial isometries $u_{1}, u_{2} \in \mathcal{N}$ such that $|T x| \leq \frac{1}{2} u_{1}^{*}\left|T\left(2 x e_{v}\right)\right| u_{1}+$ $\frac{1}{2} u_{2}^{*}\left|T\left(2 x e_{v}^{\perp}\right)\right| u_{2}$. It follows that

$$
\begin{aligned}
d(2 v ; T x) & \leq d\left(v ; \frac{1}{2} u_{1}^{*}\left|T\left(2 x e_{v}\right)\right| u_{1}\right)+d\left(v ; \frac{1}{2} u_{2}^{*}\left|T\left(2 x e_{v}^{\perp}\right)\right| u_{2}\right) \\
& \leq d\left(2 v ; T\left(2 x e_{v}\right)\right)+d\left(2 v ; T\left(2 x e_{v}^{\perp}\right)\right) .
\end{aligned}
$$

Suppose first that $q_{E}<q<\infty$. By (16) and (17) we have

$$
d(v ; T y) \leq v^{-r} C_{r}^{r}\|y\|_{L^{r}(\mathcal{M})}^{r} \quad\left(v>0, y \in L^{r}(\mathcal{M})_{+}, r=p, q\right) .
$$

Therefore,

$$
d(2 v ; T x) \leq \max \left\{C_{q}^{q}, C_{p}^{p}\right\}\left((2 v)^{-q}\left\|2 x e_{v}\right\|_{L^{q}(\mathcal{M})}^{q}+(2 v)^{-p}\left\|2 x e_{v}^{\perp}\right\|_{L^{p}(\mathcal{M})}^{p}\right),
$$

and from Proposition 4.2 it follows that

$$
\left\|x e_{v}\right\|_{L^{q}(\mathcal{M})}^{q}=\int_{\{\mu(x) \leq v\}} \mu_{t}(x)^{q} d t, \quad\left\|x e_{v}^{\perp}\right\|_{L^{p}(\mathcal{M})}^{p}=\int_{\{\mu(x)>v\}} \mu_{t}(x)^{p} d t .
$$

Therefore, by Corollary 3.6 ,

$$
\begin{aligned}
d(2 v ; T x) & \leq v^{-q} \int_{\{\mu(x) \leq v\}} \mu_{t}(x)^{q} d t+v^{-p} \int_{\{\mu(x)>v\}} \mu_{t}(x)^{p} d t \\
& =d\left(v ; \Theta_{p, q} \mu(x)\right) .
\end{aligned}
$$

The result now follows from Lemma 3.7 using that $d(v ; T x)=d(v ; \mu(T x))$. 
Suppose now that $q=\infty$. Then

$$
\left\|\frac{1}{2} u_{1}^{*} T\left(2 x e_{v}\right) u_{1}\right\|_{L^{\infty}(\mathcal{N})} \leq C_{\infty}\left\|x e_{v}\right\|_{L^{\infty}(\mathcal{M})} \leq v,
$$

so $d\left(v ; \frac{1}{2} u_{1}^{*} T\left(x e_{v}\right) u_{1}\right)=0$. By (16) and (17) we have

$$
d(v ; T y) \leq v^{-p} C_{p}^{p}\|y\|_{L^{p}(\mathcal{M})}^{p} \quad\left(v>0, y \in L^{p}(\mathcal{M})_{+}\right),
$$

and therefore (18) implies that

$$
\begin{aligned}
d(2 v ; T x) & \leq C_{p}^{p}(2 v)^{-p}\left\|2 x e_{v}^{\perp}\right\|_{L^{p}(\mathcal{M})}^{p} \\
& \leq v^{-p} \int_{\{\mu(x)>v\}} \mu_{t}(x)^{p} d t=d\left(v ; \Theta_{p, \infty} \mu(x)\right) .
\end{aligned}
$$

Lemma 3.7 gives the conclusion.

It is clear from the proof of Theorem 4.8 that the same result holds for midpoint (sub)convex operators on $L^{p}(\mathcal{M})_{h}+L^{q}(\mathcal{M})_{h}$ or $L^{p}(\mathcal{M})+L^{q}(\mathcal{M})$ instead of $L^{p}(\mathcal{M})_{+}+L^{q}(\mathcal{M})_{+}$.

The original version of Boyd's theorem allows for the interpolation of operators of weak-type $(p, p)$, i.e., which are bounded from $L^{p, 1}$ into $L^{p, \infty}$. Theorem 4.8 only applies for Marcinkiewicz weak type $(p, p)$ operators. In Theorem A.3 in the appendix we will show how to obtain a full noncommutative analogue of Boyd's theorem using a different approach.

4.1. Interpolation of noncommutative probabilistic inequalities. To illustrate the flexibility of the method used to prove Theorem 4.8, we modify it to interpolate several noncommutative probabilistic inequalities. In particular we prove the dual version of Doob's maximal inequality in noncommutative symmetric spaces; see Corollary 4.13 below. The latter result is a consequence of Theorem 4.11, which we will interpret in the following section as an interpolation result for operators on noncommutative $\ell^{1}$-valued symmetric spaces. For its proof, we shall need the following observation.

Lemma 4.10. Let $x \in S(\tau)_{+}$. If $e$ is a projection in $\mathcal{M}$, then

$$
x \leq 2\left(e x e+e^{\perp} x e^{\perp}\right) .
$$

Proof. By writing

$$
x=e x e+e^{\perp} x e+e x e^{\perp}+e^{\perp} x e^{\perp},
$$

we see that the asserted inequality is equivalent to

$$
e x e-e^{\perp} x e-e x e^{\perp}+e^{\perp} x e^{\perp} \geq 0 .
$$

But $x \geq 0$, so

$$
e x e-e^{\perp} x e-e x e^{\perp}+e^{\perp} x e^{\perp}=\left(x^{\frac{1}{2}} e-x^{\frac{1}{2}} e^{\perp}\right)^{*}\left(x^{\frac{1}{2}} e-x^{\frac{1}{2}} e^{\perp}\right) \geq 0,
$$

and the result follows.

Theorem 4.11. Let $E$ be a symmetric quasi-Banach function space on $\mathbb{R}_{+}$which is s-convex for some $0<s<\infty$. Let $\mathcal{M}, \mathcal{N}$ be von Neumann algebras equipped with normal, semi-finite, faithful traces $\tau$ and $\sigma$, respectively. Suppose that $0<p<q \leq$ $\infty$ and for every $k \geq 1$ let $T_{k}: L^{p}(\mathcal{M})_{+}+L^{q}(\mathcal{M})_{+} \rightarrow S(\sigma)_{+}$be positive midpoint 
convex maps such that for some constants $C_{p}, C_{q}>0$ depending only on $p$ and $q$, respectively,

$$
\left\|\sum_{k \geq 1} T_{k}\left(x_{k}\right)\right\|_{L^{r, \infty}(\mathcal{N})} \leq C_{r}\left\|\sum_{k \geq 1} x_{k}\right\|_{L^{r}(\mathcal{M})} \quad\left(x_{k} \in L^{r}(\mathcal{M})_{+}, k \geq 1, r=p, q\right) .
$$

If $p<p_{E} \leq q_{E}<q<\infty$ or $p<p_{E}$ and $q=\infty$, then for any sequence $\left(x_{k}\right)_{k \geq 1}$ in $E(\mathcal{M})_{+}$,

$$
\left\|\sum_{k \geq 1} T_{k}\left(x_{k}\right)\right\|_{E(\mathcal{N})} \leq 4\left\|\Theta_{p, q}\right\| \max \left\{C_{p}, C_{q}\right\}\left\|\sum_{k \geq 1} x_{k}\right\|_{E(\mathcal{M})}
$$

where the sums converge in norm.

Proof. We may assume $C_{p}, C_{q} \leq 1$. Suppose first that $q_{E}<q<\infty$. By completeness it suffices to prove (20) for a finite sequence $\left(x_{k}\right)$ in $E(\mathcal{M})_{+}$. Set $x=\sum_{k} x_{k}$. For any $v \geq 0$, let $e_{v}=e^{x}[0, v]$. By Lemma 4.10 and positivity and convexity of the $T_{k}$,

$$
\begin{aligned}
\sum_{k} T_{k}\left(x_{k}\right) & \leq \sum_{k} T_{k}\left(2 e_{v} x_{k} e_{v}+2 e_{v}^{\perp} x_{k} e_{v}^{\perp}\right) \\
& \leq \frac{1}{2} \sum_{k} T_{k}\left(4 e_{v} x_{k} e_{v}\right)+\frac{1}{2} \sum_{k} T_{k}\left(4 e_{v}^{\perp} x_{k} e_{v}^{\perp}\right) .
\end{aligned}
$$

Therefore,

$$
d\left(4 v ; \sum_{k} T_{k}\left(x_{k}\right)\right) \leq d\left(4 v ; \sum_{k} T_{k}\left(4 e_{v} x_{k} e_{v}\right)\right)+d\left(4 v ; \sum_{k} T_{k}\left(4 e_{v}^{\perp} x_{k} e_{v}^{\perp}\right)\right) .
$$

By (19),

$$
\begin{aligned}
d\left(4 v ; \sum_{k} T_{k}\left(x_{k}\right)\right) & \leq(4 v)^{-q}\left\|\sum_{k} 4 e_{v} x_{k} e_{v}\right\|_{L^{q}(\mathcal{M})}^{q}+(4 v)^{-p}\left\|\sum_{k} 4 e_{v}^{\perp} x_{k} e_{v}^{\perp}\right\|_{L^{p}(\mathcal{M})}^{p} \\
& =v^{-q} \int_{\{\mu(x) \leq v\}} \mu_{t}(x)^{q} d t+v^{-p} \int_{\{\mu(x)>v\}} \mu_{t}(x)^{p} d t \\
& =d\left(v ; \Theta_{p, q} \mu(x)\right),
\end{aligned}
$$

where the final equality follows from Corollary [3.6. The result is now immediate from Lemma 3.7. The case $q=\infty$ follows analogously as in the proof of Theorem 4.8

As an application of Theorem 4.11, we can interpolate the following dual Doob inequality in noncommutative $L^{p}$-spaces, due to M. Junge.

Theorem 4.12 ([17]). Let $\mathcal{M}$ be a semi-finite von Neumann algebra and let $\left(\mathcal{E}_{k}\right)_{k \geq 1}$ be an increasing sequence of conditional expectations in $\mathcal{M}$. If $1 \leq p<\infty$, then for any sequence $\left(x_{k}\right)_{k \geq 1}$ in $L^{p}(\mathcal{M})_{+}$,

$$
\left\|\sum_{k} \mathcal{E}_{k}\left(x_{k}\right)\right\|_{L^{p}(\mathcal{M})} \lesssim p\left\|\sum_{k} x_{k}\right\|_{L^{p}(\mathcal{M})} .
$$

Theorems 4.12 and 4.11 together yield the following extension.

Corollary 4.13. Let $E$ be a symmetric quasi-Banach function space on $\mathbb{R}_{+}$which is s-convex for some $0<s<\infty$ and let $\mathcal{M}$ be a semi-finite von Neumann algebra. 
Let $\left(\mathcal{E}_{k}\right)_{k \geq 1}$ be an increasing sequence of conditional expectations in $\mathcal{M}$. If $1<$ $p_{E} \leq q_{E}<\infty$, then for any sequence $\left(x_{k}\right)_{k \geq 1}$ in $E(\mathcal{M})_{+}$,

$$
\left\|\sum_{k \geq 1} \mathcal{E}_{k}\left(x_{k}\right)\right\|_{E(\mathcal{M})} \lesssim_{E}\left\|\sum_{k \geq 1} x_{k}\right\|_{E(\mathcal{M})}
$$

where the sums converge in norm.

In 18, Theorem 7.1, it was shown that any conditional expectation $\mathcal{E}$ is 'antibounded' for the $L^{p}$-norm if $0<p<1$, i.e.,

$$
\|x\|_{L^{p}(\mathcal{M})} \leq 2^{\frac{1}{p}}\|\mathcal{E}(x)\|_{L^{p}(\mathcal{M})} \quad(x \in \mathcal{M}) .
$$

Even though (22) does not correspond to the boundedness of an operator, we can still 'interpolate' this estimate.

Proposition 4.14. Let $\mathcal{M}$ be a finite von Neumann algebra and let $\mathcal{E}$ be a conditional expectation on $\mathcal{M}$. If $E$ is a symmetric quasi-Banach function space on $\mathbb{R}_{+}$ with $q_{E}<1$, then

$$
\|x\|_{E(\mathcal{M})} \lesssim_{E}\|\mathcal{E}(x)\|_{E(\mathcal{M})} \quad(x \in \mathcal{M}) .
$$

Proof. Let $y=\mathcal{E}(x)$ and for $v>0$ set $e_{v}=e^{y}[0, v]$. As was remarked after Lemma 2.2, we have $p_{E}>0$ and hence we can fix $0<p<p_{E}$ and $q_{E}<q<1$. By Chebyshev's inequality and (22),

$$
\begin{aligned}
d\left(2^{1+\frac{1}{p}} v ; x\right) & \leq v^{-q}\left\|2^{-\frac{1}{p}} x e_{v}\right\|_{L^{q}(\mathcal{M})}^{q}+v^{-p}\left\|2^{-\frac{1}{p}} x e_{v}^{\perp}\right\|_{L^{p}(\mathcal{M})}^{p} \\
& \leq v^{-q}\left\|\mathcal{E}\left(x e_{v}\right)\right\|_{L^{q}(\mathcal{M})}^{q}+v^{-p}\left\|\mathcal{E}\left(x e_{v}^{\perp}\right)\right\|_{L^{p}(\mathcal{M})}^{p} \\
& =v^{-q}\left\|\mathcal{E}(x) e_{v}\right\|_{L^{q}(\mathcal{M})}^{q}+v^{-p}\left\|\mathcal{E}(x) e_{v}^{\perp}\right\|_{L^{p}(\mathcal{M})}^{p} \\
& =v^{-q} \int_{\{\mu(y) \leq v\}} \mu_{t}(y)^{q} d t+v^{-p} \int_{\{\mu(y)>v\}} \mu_{t}(y)^{p} d t \\
& =d\left(v ; \Theta_{p, q} \mu(y)\right),
\end{aligned}
$$

where the final equality follows from Corollary 3.6. The conclusion now follows from Lemma 3.7

The following result facilitates interpolation of noncommutative square function estimates.

Theorem 4.15. Let $E$ be a symmetric quasi-Banach function space on $\mathbb{R}_{+}$which is s-convex for some $0<s<\infty$. Let $\mathcal{M}, \mathcal{N}$ be von Neumann algebras equipped with normal, semi-finite, faithful traces $\tau$ and $\sigma$, respectively. Suppose that $0<p<$ $q \leq \infty$ and for $k \geq 1$ let $T_{k}: L^{p}(\mathcal{M})+L^{q}(\mathcal{M}) \rightarrow S(\sigma)$ be linear maps such that for some constants $C_{p}, C_{q}>0$ depending only on $p$ and $q$, respectively,

$$
\begin{aligned}
& \left\|\sum_{k \geq 1} T_{k}\left(x_{k}\right)\right\|_{L^{r, \infty}(\mathcal{N})} \\
& \quad \leq C_{r}\left\|\left(\sum_{k \geq 1}\left|x_{k}\right|^{2}\right)^{\frac{1}{2}}\right\|_{L^{r}(\mathcal{M})} \quad\left(x_{k} \in L^{r}(\mathcal{M})_{+}, k \geq 1, r=p, q\right) .
\end{aligned}
$$

If $p<p_{E} \leq q_{E}<q<\infty$ or $p<p_{E}$ and $q=\infty$, then for any finite sequence $\left(x_{k}\right)$ in $E(\mathcal{M})$,

$$
\left\|\sum_{k \geq 1} T_{k}\left(x_{k}\right)\right\|_{E(\mathcal{N})} \leq 2\left\|\Theta_{p, q}\right\| \max \left\{C_{p}, C_{q}\right\}\left\|\left(\sum_{k \geq 1}\left|x_{k}\right|^{2}\right)^{\frac{1}{2}}\right\|_{E(\mathcal{M})} .
$$


Proof. We may assume $C_{p}, C_{q} \leq 1$. Let $x=\left(\sum_{k \geq 1}\left|x_{k}\right|^{2}\right)^{\frac{1}{2}}$ and for $v>0$ define $e_{v}=e^{x}[0, v]$. Then,

$$
d\left(2 v ; \sum_{k \geq 1} T_{k}\left(x_{k}\right)\right) \leq d\left(v ; \sum_{k \geq 1} T_{k}\left(x_{k} e_{v}^{\perp}\right)\right)+d\left(v ; \sum_{k \geq 1} T_{k}\left(x_{k} e_{v}\right)\right) .
$$

By (23),

$$
\begin{aligned}
d\left(2 v ; \sum_{k \geq 1} T_{k}\left(x_{k}\right)\right) & \leq v^{-p}\left\|\left(\sum_{k \geq 1}\left|x_{k} e_{v}^{\perp}\right|^{2}\right)^{\frac{1}{2}}\right\|_{L^{p}(\mathcal{M})}^{p}+v^{-q}\left\|\left(\sum_{k \geq 1}\left|x_{k} e_{v}\right|^{2}\right)^{\frac{1}{2}}\right\|_{L^{q}(\mathcal{M})}^{q} \\
& =v^{-p}\left\|\left(\sum_{k \geq 1}\left|x_{k}\right|^{2}\right)^{\frac{1}{2}} e_{v}^{\perp}\right\|_{L^{p}(\mathcal{M})}^{p}+v^{-q}\left\|\left(\sum_{k \geq 1}\left|x_{k}\right|^{2}\right)^{\frac{1}{2}} e_{v}\right\|_{L^{q}(\mathcal{M})}^{q} \\
& =v^{-p} \int_{\{\mu(x)>v\}} \mu_{t}(x)^{p} d t+v^{-q} \int_{\{\mu(x) \leq v\}} \mu_{t}(x)^{q} d t \\
& =d\left(v ; \Theta_{p, q} \mu(x)\right) .
\end{aligned}
$$

The result now follows from Lemma 3.7. The case $q=\infty$ is similar.

As a corollary, we find the following version of Stein's inequality for noncommutative symmetric spaces, which will be needed in the proof of Theorem 6.2 below. A different proof of this result was found in [16].

Corollary 4.16. Let $E$ be a symmetric quasi-Banach function space on $\mathbb{R}_{+}$which is $s$-convex for some $0<s<\infty$ and let $\mathcal{M}$ be a semi-finite von Neumann algebra. Let $\left(\mathcal{E}_{k}\right)_{k \geq 1}$ be an increasing sequence of conditional expectations in $\mathcal{M}$. If $1<$ $p_{E} \leq q_{E}<\infty$, then for any finite sequence $\left(x_{k}\right)$ in $E(\mathcal{M})$,

$$
\left\|\left(\sum_{k \geq 1}\left|\mathcal{E}_{k}\left(x_{k}\right)\right|^{2}\right)^{\frac{1}{2}}\right\|_{E(\mathcal{M})} \lesssim_{E}\left\|\left(\sum_{k \geq 1}\left|x_{k}\right|^{2}\right)^{\frac{1}{2}}\right\|_{E(\mathcal{M})} .
$$

Proof. Let $e_{k l}$ be the standard matrix units, let $y_{k}=x_{k} \otimes e_{k 1}$ and let $T_{k}=\mathcal{E}_{k} \otimes$ $\mathbf{1}_{B\left(\ell^{2}\right)}$. Then (24) is equivalent to

$$
\left\|\sum_{k \geq 1} T_{k}\left(y_{k}\right)\right\|_{E\left(\mathcal{M} \otimes B\left(\ell^{2}\right)\right)} \lesssim_{E}\left\|\left(\sum_{k \geq 1}\left|y_{k}\right|^{2}\right)^{\frac{1}{2}}\right\|_{E\left(\mathcal{M} \otimes B\left(\ell^{2}\right)\right)} .
$$

By [32], Theorem 2.3, this inequality holds if $E=L^{p}$ with $1<p<\infty$. Hence, the result follows immediately from Theorem 4.15

Further examples of probabilistic inequalities which can be interpolated using the presented method are given by the 'upper' noncommutative Khintchine inequalities; see [7, Theorem 4.1, and [8], Corollary 2.2.

Remark 4.17. The results in Theorems 4.8, 4.11 and 4.15, Corollaries 4.13 and 4.16, and Proposition 4.14 all have an appropriate ' $\Phi$-moment version'. Indeed, these versions follow immediately by using Lemma 3.9 instead of Lemma 3.7 in the proofs of the latter results. In particular, by following the proof of Theorem 4.8 and taking Remark 3.10 into account, we find an extension of [1], Theorem 2.1, for nonconvex Orlicz functions. 


\section{INTERPOLATION OF NONCOMMUTATIVE MAXIMAL INEQUALITIES}

In this section we present a Boyd-type interpolation theorem for noncommutative maximal inequalities. To formulate maximal inequalities in noncommutative symmetric spaces and their dual versions, we first introduce the two 'noncommutative vector-valued symmetric spaces' $E\left(\mathcal{M} ; \ell^{\infty}\right)$ and $E\left(\mathcal{M} ; \ell^{1}\right)$. We define these in analogy with the noncommutative vector-valued $L^{p}$-spaces $L^{p}\left(\mathcal{M} ; \ell^{\infty}\right)$ and $L^{p}\left(\mathcal{M} ; \ell^{1}\right)$, which were introduced in 31 for hyperfinite von Neumann algebras and considered in general in [17. From now on, we let $E$ be a symmetric Banach function space on $\mathbb{R}_{+}$.

We define $E\left(\mathcal{M} ; \ell^{\infty}\right)$ to be the space of all sequences $x=\left(x_{k}\right)_{k \geq 1}$ in $E(\mathcal{M})$ for which there exist $a, b \in E^{(2)}(\mathcal{M})$ and a bounded sequence $y=\left(y_{k}\right)_{k \geq 1}$ such that

$$
x_{k}=a y_{k} b \quad(k \geq 1) .
$$

For $x \in E\left(\mathcal{M} ; \ell^{\infty}\right)$ we define

$$
\|x\|_{E\left(\mathcal{M} ; \ell^{\infty}\right)}=\inf \left\{\|a\|_{E^{(2)}(\mathcal{M})} \sup _{k \geq 1}\left\|y_{k}\right\|_{\infty}\|b\|_{E^{(2)}(\mathcal{M})}\right\},
$$

where the infimum is taken over all possible factorizations of $x$ as above. We can think of the quantity (25) as ' $\left\|\sup _{k \geq 1} x_{k}\right\|_{E(\mathcal{M})}$ ', even though $\sup _{k \geq 1} x_{k}$ need not be defined at all.

We define $E\left(\mathcal{M} ; \ell^{1}\right)$ to be the space of all sequences $x=\left(x_{k}\right)_{k \geq 1}$ in $E(\mathcal{M})$ which can be decomposed as

$$
x_{k}=\sum_{j \geq 1} u_{j k}^{*} v_{j k} \quad(k \geq 1)
$$

for two families $\left(u_{j k}\right)_{j, k \geq 1}$ and $\left(v_{j k}\right)_{j, k \geq 1}$ in $E^{(2)}(\mathcal{M})$ satisfying

$$
\sum_{j, k} u_{j k}^{*} u_{j k} \in E(\mathcal{M}) \text { and } \sum_{j, k} v_{j k}^{*} v_{j k} \in E(\mathcal{M}),
$$

where the series converge in norm. For $x \in E\left(\mathcal{M} ; \ell^{1}\right)$ we define

$$
\|x\|_{E\left(\mathcal{M} ; \ell^{1}\right)}=\inf \left\{\left\|\sum_{j, k} u_{j k}^{*} u_{j k}\right\|_{E(\mathcal{M})}^{\frac{1}{2}}\left\|\sum_{j, k} v_{j k}^{*} v_{j k}\right\|_{E(\mathcal{M})}^{\frac{1}{2}}\right\},
$$

where the infimum is taken over all decompositions of $x$ as above. In what follows, we will mostly consider elements $x=\left(x_{k}\right)_{k \geq 1} \in E\left(\mathcal{M} ; \ell^{1}\right)$ for which $x_{k} \geq 0$ for all $k$. In this case,

$$
\|x\|_{E\left(\mathcal{M} ; \ell^{1}\right)}=\left\|\sum_{k \geq 1} x_{k}\right\|_{E(\mathcal{M})} .
$$

The theory for the spaces $E\left(\mathcal{M} ; \ell^{\infty}\right)$ and $E\left(\mathcal{M} ; \ell^{1}\right)$ can be developed in full analogy with the special case $E=L^{p}$ considered in [17, 19, 34. In fact, most of the basic results follow verbatim as soon as we replace $L^{p}$ by $E, L^{p^{\prime}}$ by $E^{\times}$, where $\frac{1}{p}+\frac{1}{p^{\prime}}=$ 1 , and $L^{2 p}$ by $E^{(2)}$ in the proofs of these results. For example, the following observation is immediate.

Theorem 5.1. If $E$ is a symmetric Banach function space on $\mathbb{R}_{+}$, then $E\left(\mathcal{M} ; \ell^{\infty}\right)$ and $E\left(\mathcal{M} ; \ell^{1}\right)$ are Banach spaces.

Our strategy to prove a Boyd-type interpolation theorem for maximal inequalities is to dualize Theorem 4.11, which can be viewed as a Boyd-type interpolation theorem for $\ell^{1}$-valued noncommutative symmetric spaces. We shall need the duality 
stated in Theorem 5.3 below. The proof is essentially an adaptation of the HahnBanach separation argument in [17], Proposition 3.6 (see also [34, Theorem 4.11) to our context. We need the following observation, proved in [11, Theorem 5.6 and p. 745 .

Theorem 5.2. If $E$ is a separable symmetric Banach function space on $\mathbb{R}_{+}$, then $E(\mathcal{M})^{*}=E^{\times}(\mathcal{M})$ isometrically, with associated duality bracket given by

$$
\langle x, y\rangle=\tau(x y) \quad\left(x \in E(\mathcal{M}), y \in E^{\times}(\mathcal{M})\right) .
$$

Below we will implicitly use the trace property a number of times; i.e., we will use that if $x, y \in S(\tau)$ are such that $x y, y x \in L^{1}(\mathcal{M})$, then $\tau(x y)=\tau(y x)$. In particular this holds if $x \in E(\mathcal{M})$ and $y \in E^{\times}(\mathcal{M})$.

Theorem 5.3. Let $\mathcal{M}$ be a semi-finite von Neumann algebra and let $E$ be a separable symmetric Banach function space on $\mathbb{R}_{+}$. If $y=\left(y_{k}\right) \in E^{\times}\left(\mathcal{M} ; \ell^{\infty}\right)$ satisfies $y_{k} \geq 0$ for all $k$, then

$$
\|y\|_{E^{\times}\left(\mathcal{M} ; \ell^{\infty}\right)}=\sup \left\{\sum_{k \geq 1} \tau\left(x_{k} y_{k}\right): x_{k} \in E(\mathcal{M})_{+},\left\|\sum_{k \geq 1} x_{k}\right\|_{E(\mathcal{M})} \leq 1\right\} .
$$

Proof. We let $S$ denote the supremum on the right hand side of (26). Let $y_{k}=a z_{k} b$ with $a, b \in\left(E^{\times}\right)^{(2)}(\mathcal{M})$ and $z_{k} \in \mathcal{M}$ with $\left\|z_{k}\right\|_{\infty} \leq 1$ and let $\left(x_{k}\right)$ be a sequence in $E(\mathcal{M})_{+}$. By Hölder's inequality,

$$
\begin{aligned}
\sum_{k} \tau\left(x_{k} y_{k}\right) & =\sum_{k} \tau\left(x_{k} a z_{k} b\right)=\sum_{k} \tau\left(b x_{k}^{\frac{1}{2}} x_{k}^{\frac{1}{2}} a z_{k}\right) \\
& \leq \sum_{k}\left\|b x_{k}^{\frac{1}{2}}\right\|_{L^{2}(\mathcal{M})}\left\|x_{k}^{\frac{1}{2}} a z_{k}\right\|_{L^{2}(\mathcal{M})} \\
& \leq\left(\sum_{k} \tau\left(b x_{k} b^{*}\right)\right)^{\frac{1}{2}}\left(\sum_{k} \tau\left(a^{*} x_{k} a\right)\right)^{\frac{1}{2}} \\
& \leq\|b\|_{\left(E^{\times}\right)^{(2)}(\mathcal{M})}\left\|\sum_{k} x_{k}\right\|_{E(\mathcal{M})}\|a\|_{\left(E^{\times}\right)^{(2)}(\mathcal{M})} .
\end{aligned}
$$

We conclude that $S \leq\|y\|_{E^{\times}\left(\mathcal{M} ; \ell^{\infty}\right)}$.

Suppose now that $S=1$; we will show that $\|y\|_{E^{\times}\left(\mathcal{M} ; \ell^{\infty}\right)} \leq 1$. Under this assumption, we have for any finite sequence $x=\left(x_{k}\right)$ in $E(\mathcal{M})_{+}$,

$$
\sum_{k} \tau\left(x_{k} y_{k}\right) \leq\left\|\sum_{k} x_{k}\right\|_{E(\mathcal{M})}
$$

Let $K=\left\{s \in E^{\times}(\mathcal{M})_{+}:\|s\|_{E^{\times}(\mathcal{M})} \leq 1\right\}$, equipped with the weak ${ }^{*}$ topology of $E(\mathcal{M})^{*}$. Since $E^{\times}(\mathcal{M})$ is isometrically isomorphic to $E(\mathcal{M})^{*}$ by Theorem 5.2 and $E^{\times}(\mathcal{M})_{+}$is weak ${ }^{*}$ closed in $E^{\times}(\mathcal{M})$, we conclude that $K$ is compact by the Banach-Alaoglu theorem. Moreover,

$$
\|w\|_{E(\mathcal{M})}=\sup _{s \in K} \tau(w s) \quad\left(w \in E(\mathcal{M})_{+}\right) .
$$

For any finite sequence $x$ as above we define

$$
f_{x}(s)=\sum_{k} \tau\left(x_{k} s\right)-\sum_{k} \tau\left(x_{k} y_{k}\right) .
$$

Clearly $f_{x}$ is a real-valued continuous function on $K$, and from (27) it follows that $\sup _{s \in K} f_{x}(s) \geq 0$. Let $A$ be the subset of $C(K)$ consisting of all $f_{x}$, where 
$x=\left(x_{k}\right)$ is a finite sequence in $E(\mathcal{M})_{+}$. Then $A$ is a cone in $C(K)$. Indeed, if $\lambda \geq 0$, then $\lambda f_{x}=f_{\lambda x}$. Moreover, if $x, \tilde{x}$ are finite families in $E(\mathcal{M})$, then $f_{x}+f_{\tilde{x}}$ can be realized as $f_{x+\tilde{x}}$, since without loss of generality we may assume that $\tilde{x}$ is to the right of the finite family $x$. Observe that $A$ is disjoint from the cone $A_{-}=\{g \in C(K): \sup g<0\}$. By the Hahn-Banach separation theorem, there exists a real Borel measure $\mu$ on $K$ and $\alpha \in \mathbb{R}$ such that for all $f \in A$ and $g \in A_{-}$,

$$
\int_{K} g d \mu \leq \alpha \leq \int_{K} f d \mu .
$$

Note that $\alpha=0$, as both $A$ and $A_{-}$are cones. If $B$ is a Borel subset, then we can find a sequence $\left(g_{i}\right)$ in $A_{-}$such that $g_{n} \uparrow-\chi_{B}$. This shows that $\mu$ must be positive, and by normalization we may assume that $\mu$ is a probability measure. Hence, for all $k \geq 1$, we have

$$
\tau\left(x y_{k}\right) \leq \int_{K} \tau(x s) d \mu(s) \quad\left(x \in E(\mathcal{M})_{+}\right) .
$$

Define a positive operator by

$$
a=\int_{K} s d \mu(s)
$$

Clearly $a \in K$, so $a \in E^{\times}(\mathcal{M})_{+}$and $\|a\|_{E^{\times}(\mathcal{M})} \leq 1$. By (28) and normality of $\tau$,

$$
\tau\left(x y_{k}\right) \leq \tau(x a) \quad\left(x \in E(\mathcal{M})_{+}\right) .
$$

This implies that $y_{k} \leq a$, and therefore we find a contraction $u_{k} \in \mathcal{M}$ such that $y_{k}^{\frac{1}{2}}=u_{k} a^{\frac{1}{2}}$. In particular, $y_{k}=a^{\frac{1}{2}} u_{k}^{*} u_{k} a^{\frac{1}{2}}$ and hence

$$
\|y\|_{E^{\times}(\mathcal{M} ; \ell \infty)} \leq\|a\|_{E^{\times}(\mathcal{M})} \leq 1 .
$$

This completes the proof.

Remark 5.4. Using a slightly more involved version of the separation argument in the proof of Theorem 5.3 (see the proof of [17], Proposition 3.6, for the case $\left.E=L^{p}\right)$, one may show that in fact

$$
E\left(\mathcal{M} ; \ell^{1}\right)^{*}=E^{\times}\left(\mathcal{M} ; \ell^{\infty}\right)
$$

isometrically, with respect to the duality bracket

$$
\langle x, y\rangle=\sum_{k \geq 1} \tau\left(x_{k} y_{k}\right)
$$

where $x \in E\left(\mathcal{M} ; \ell^{1}\right)$ and $y \in E^{\times}\left(\mathcal{M} ; \ell^{\infty}\right)$.

The following result facilitates the interpolation of noncommutative maximal inequalities.

Theorem 5.5. Let $E$ be the Köthe dual of a separable symmetric Banach function space on $\mathbb{R}_{+}$. Suppose that $1 \leq p<q<\infty$ and let $S_{k}: L^{p, 1}(\mathcal{M})_{+}+L^{q, 1}(\mathcal{M})_{+} \rightarrow$ $S(\tau)_{+}$be positive linear operators satisfying

$$
\left\|\left(S_{k}(x)\right)_{k \geq 1}\right\|_{L^{r}\left(\mathcal{M} ; \ell^{\infty}\right)} \lesssim_{r}\|x\|_{L^{r, 1}(\mathcal{M})} \quad\left(x \in L^{r, 1}(\mathcal{M})_{+}, r=p, q\right) .
$$

If $q_{E}<q$ and either $p=1$ or $p_{E}>p$, then

$$
\left\|\left(S_{k}(x)\right)_{k \geq 1}\right\|_{E\left(\mathcal{M} ; \ell^{\infty}\right)} \lesssim_{E}\|x\|_{E(\mathcal{M})} \quad\left(x \in E(\mathcal{M})_{+}\right) .
$$


Proof. Let $F$ be the symmetric space on $\mathbb{R}_{+}$such that $F^{\times}=E$. By (5) we have $p_{F}>q^{\prime}$, and if $p_{E}>1$ also $q_{F}<p^{\prime}$. Since $S_{k}$ is positive, so is its adjoint $S_{k}^{*}$. If $r \in\{p, q\}$ and $y=\left(y_{k}\right) \in L^{r^{\prime}}\left(\mathcal{M} ; \ell^{1}\right)$ with $y_{k} \geq 0$, then for any $x \in L^{r, 1}(\mathcal{M})_{+}$,

$$
\begin{aligned}
\sum_{k \geq 1} \tau\left(S_{k}^{*}\left(y_{k}\right) x\right) & =\sum_{k \geq 1} \tau\left(y_{k} S_{k}(x)\right) \\
& \leq\|y\|_{L^{r^{\prime}}\left(\mathcal{M} ; \ell^{1}\right)}\left\|\left(S_{k}(x)\right)_{k \geq 1}\right\|_{L^{r}\left(\mathcal{M} ; \ell^{\infty}\right)} \\
& \lesssim r r\|y\|_{L^{r^{\prime}}\left(\mathcal{M} ; \ell^{1}\right)}\|x\|_{L^{r, 1}(\mathcal{M})}
\end{aligned}
$$

It follows that

$$
\left\|\sum_{k \geq 1} S_{k}^{*}\left(y_{k}\right)\right\|_{L^{r^{\prime}, \infty}(\mathcal{M})} \lesssim p\left\|\sum_{k \geq 1} y_{k}\right\|_{L^{r^{\prime}}(\mathcal{M})} .
$$

Therefore, if $\left(y_{k}\right) \in F\left(\mathcal{M} ; \ell^{1}\right)$ satisfies $y_{k} \geq 0$, then by Theorem 4.11,

$$
\left\|\sum_{k \geq 1} S_{k}^{*}\left(y_{k}\right)\right\|_{F(\mathcal{M})} \lesssim_{F}\left\|\sum_{k \geq 1} y_{k}\right\|_{F(\mathcal{M})} .
$$

Hence, if $x \in E(\mathcal{M})_{+}$, then

$$
\begin{aligned}
\sum_{k \geq 1} \tau\left(y_{k} S_{k}(x)\right) & =\sum_{k \geq 1} \tau\left(S_{k}^{*}\left(y_{k}\right) x\right) \\
& \leq\left\|\sum_{k \geq 1} S_{k}^{*}\left(y_{k}\right)\right\|_{F(\mathcal{M})}\|x\|_{E(\mathcal{M})} \lesssim_{E}\left\|\sum_{k \geq 1} y_{k}\right\|_{F(\mathcal{M})}\|x\|_{E(\mathcal{M})} .
\end{aligned}
$$

By Theorem 5.3 we conclude that (30) holds.

Examples of sequences of operators satisfying the conditions of Theorem 5.5 are established in [19. We give two examples which yield maximal ergodic inequalities in noncommutative symmetric spaces. Let $T: \mathcal{M} \rightarrow \mathcal{M}$ be a linear map such that

(a) $T$ is a contraction on $\mathcal{M}$;

(b) $T$ is positive;

(c) $\tau(T(x)) \leq \tau(x)$ for all $x \in L^{1}(\mathcal{M}) \cap \mathcal{M}_{+}$.

In [19], Theorem 4.1 , it is shown that for any $1<p \leq \infty$ the ergodic averages

$$
M_{k}(T)=\frac{1}{k+1} \sum_{i=0}^{k} T^{i} \quad(k \geq 1)
$$

satisfy the maximal inequality

$$
\left\|\left(M_{k}(T)(x)\right)_{k \geq 1}\right\|_{L^{p}\left(\mathcal{M} ; \ell^{\infty}\right)} \lesssim_{p}\|x\|_{L^{p}(\mathcal{M})} \quad\left(x \in L^{p}(\mathcal{M})_{+}\right) .
$$

If $T$ moreover satisfies

(d) $\tau\left(T(y)^{*} x\right)=\tau\left(y^{*} T(x)\right)$ for all $x, y \in L^{2}(\mathcal{M}) \cap \mathcal{M}$, then, as was observed in [19], Theorem 5.1 , for every $1<p \leq \infty$ one has

$$
\left\|\left(T^{k}(x)\right)_{k \geq 1}\right\|_{L^{p}\left(\mathcal{M} ; \ell^{\infty}\right)} \lesssim p\|x\|_{L^{p}(\mathcal{M})} \quad\left(x \in L^{p}(\mathcal{M})_{+}\right) .
$$

Using Theorem [5.5 we can interpolate these inequalities to obtain the following result.

Theorem 5.6. Let $E$ be the Köthe dual of a separable symmetric Banach function space on $\mathbb{R}_{+}$and suppose that $1<p_{E} \leq q_{E}<\infty$. If $T: \mathcal{M} \rightarrow \mathcal{M}$ is a linear operator satisfying conditions (a)-(c) above, then

$$
\left\|\left(M_{k}(T)(x)\right)_{k \geq 1}\right\|_{E\left(\mathcal{M} ; \ell^{\infty}\right)} \lesssim_{E}\|x\|_{E(\mathcal{M})} \quad\left(x \in E(\mathcal{M})_{+}\right) .
$$


If $T$ moreover satisfies condition (d), then

$$
\left\|\left(T^{k}(x)\right)_{k \geq 1}\right\|_{E\left(\mathcal{M} ; \ell^{\infty}\right)} \lesssim_{E}\|x\|_{E(\mathcal{M})} \quad\left(x \in E(\mathcal{M})_{+}\right) .
$$

To conclude this section, we prove a version of Doob's maximal inequality for noncommutative symmetric spaces. First recall the following definitions. Let $E$ be a symmetric Banach function space on $\mathbb{R}_{+}$and let $\mathcal{M}$ be a semi-finite von Neumann algebra. Suppose that $\left(\mathcal{M}_{k}\right)_{k \geq 1}$ is a filtration, i.e., an increasing sequence of von Neumann subalgebras such that $\left.\tau\right|_{\mathcal{M}_{k}}$ is semi-finite, and let $\mathcal{E}_{k}$ be the conditional expectation with respect to $\mathcal{M}_{k}$. A sequence $\left(y_{k}\right)$ in $E(\mathcal{M})$ is called a martingale with respect to $\left(\mathcal{M}_{k}\right)$ if $\mathcal{E}_{k}\left(y_{k+1}\right)=y_{k}$ for all $k \geq 1$. We say that $\left(y_{k}\right)$ is finite if there is an $n \geq 1$ such that $y_{k}=y_{n}$ for all $k \geq n$. A sequence $\left(x_{k}\right)$ in $E(\mathcal{M})$ is called a martingale difference sequence if $x_{k}=y_{k}-y_{k-1}$ for some martingale $\left(y_{k}\right)$, with the convention $y_{0}=0$ and $\mathcal{M}_{0}=\mathbb{C} 1$.

It was shown by M. Junge in [17] that for every $1<p \leq \infty$,

$$
\left\|\left(\mathcal{E}_{k}(y)\right)_{k \geq 1}\right\|_{L^{p}(\mathcal{M} ; \ell \infty)} \lesssim p\|y\|_{L^{p}(\mathcal{M})} .
$$

This result implies the following version for noncommutative symmetric spaces.

Theorem 5.7. Let $\mathcal{M}$ be a semi-finite von Neumann algebra and let $E$ be the Köthe dual of a separable symmetric Banach function space on $\mathbb{R}_{+}$with $1<p_{E} \leq q_{E}<\infty$. For any $y \in E(\mathcal{M})$ and any increasing sequence of conditional expectations $\left(\mathcal{E}_{k}\right)_{k \geq 1}$,

$$
\left\|\left(\mathcal{E}_{k}(y)\right)_{k \geq 1}\right\|_{E\left(\mathcal{M} ; \ell^{\infty}\right)} \lesssim_{E}\|y\|_{E(\mathcal{M})} .
$$

If $\left(y_{k}\right)_{k \geq 1}$ is a martingale in $E(\mathcal{M})$, then

$$
\sup _{k \geq 1}\left\|y_{k}\right\|_{E(\mathcal{M})} \leq\left\|\left(y_{k}\right)_{k \geq 1}\right\|_{E(\mathcal{M} ; \ell)} \lesssim_{E} \sup _{k \geq 1}\left\|y_{k}\right\|_{E(\mathcal{M})} .
$$

Proof. The first statement follows immediately from Theorem [5.5] and (31). To prove the second statement, let $y_{k}=a z_{k} b$ with $\left(z_{k}\right)_{k \geq 1}$ a bounded sequence in $\mathcal{M}$ and $a, b \in E^{(2)}(\mathcal{M})$. Since $E$ is the Köthe dual of a symmetric space, it has the Fatou property and is hence fully symmetric. Therefore, $\mu\left(y_{k}\right) \prec \prec \mu\left(a z_{k}\right) \mu(b)$ implies that

$$
\begin{aligned}
\left\|y_{k}\right\|_{E(\mathcal{M})} & \leq\left\|\mu\left(a z_{k}\right) \mu(b)\right\|_{E} \\
& \leq\left\|a z_{k}\right\|_{E^{(2)}(\mathcal{M})}\|b\|_{E^{(2)}(\mathcal{M})} \\
& \leq\|a\|_{E^{(2)}(\mathcal{M})}\left\|z_{k}\right\|_{\infty}\|b\|_{E^{(2)}(\mathcal{M})}
\end{aligned}
$$

Taking the infimum over all decompositions as above gives

$$
\sup _{k \geq 1}\left\|y_{k}\right\|_{E(\mathcal{M})} \leq\left\|\left(y_{k}\right)_{k \geq 1}\right\|_{E\left(\mathcal{M} ; \ell^{\infty}\right)} .
$$

For the reverse inequality, observe that $E(\mathcal{M}) \subset L^{p}(\mathcal{M})+L^{q}(\mathcal{M})$ for some $1<p<$ $p_{E} \leq q_{E}<q<\infty$. Let $\left(y_{k}\right)_{k \geq 1}$ be a martingale in $E(\mathcal{M})$ with $\sup _{k \geq 1}\left\|y_{k}\right\|_{E(\mathcal{M})}=$ 1. Then $\left(y_{k}\right)$ is a bounded martingale in $L^{p}(\mathcal{M})+L^{q}(\mathcal{M})$, and hence there exists $y_{\infty} \in L^{p}(\mathcal{M})+L^{q}(\mathcal{M})$ such that $y_{k} \rightarrow y_{\infty}$ in $L^{p}(\mathcal{M})+L^{q}(\mathcal{M})$ and $\mathcal{E}_{k}\left(y_{\infty}\right)=y_{k}$ for all $k \geq 1$. Since $E(\mathcal{M})$ has the Fatou property, its unit ball is closed in $S(\tau)$ (cf. 11], Proposition 5.14). As $y_{k} \rightarrow y_{\infty}$ in measure, we conclude that $y_{\infty} \in E(\mathcal{M})$ and $\left\|y_{\infty}\right\|_{E(\mathcal{M})} \leq 1$. Applying (32) for $y=y_{\infty}$ yields the result.

Remark 5.8. While the author was completing this paper, a preprint 2] appeared in which Theorems 5.6 and 5.7 are proved (under slightly different assumptions) by an alternative method. 


\section{Burkholder-Davis-Gundy and Burkholder-Rosenthal inequalities}

As applications of the noncommutative version of Doob's maximal inequality and its dual version, we derive versions of the Burkholder-Davis-Gundy inequalities and Burkholder-Rosenthal inequalities in noncommutative symmetric spaces. Let $E$ be a symmetric Banach function space on $\mathbb{R}_{+}$. For any finite martingale difference sequence $\left(x_{k}\right)$ in $E(\mathcal{M})$ we set

$$
\left\|\left(x_{k}\right)\right\|_{H_{c}^{E}}=\left\|\left(\sum_{k}\left|x_{k}\right|^{2}\right)^{\frac{1}{2}}\right\|_{E(\mathcal{M})} ;\left\|\left(x_{k}\right)\right\|_{H_{r}^{E}}=\left\|\left(\sum_{k}\left|x_{k}^{*}\right|^{2}\right)^{\frac{1}{2}}\right\|_{E(\mathcal{M})} .
$$

These expressions define two norms on the linear space of all finite martingale difference sequences in $E(\mathcal{M})$. The following Burkholder-Davis-Gundy inequalities extend the Burkholder-Gundy inequalities established in [7].

Theorem 6.1. Let $E$ be the Köthe dual of a separable symmetric Banach function space on $\mathbb{R}_{+}$and suppose that $1<p_{E} \leq q_{E}<\infty$. Let $\mathcal{M}$ be a semi-finite von Neumann algebra and $\left(\mathcal{M}_{k}\right)_{k \geq 1}$ a filtration in $\mathcal{M}$. Then, for any martingale difference sequence $\left(x_{k}\right)$ of a finite martingale $\left(y_{k}\right)$ in $E(\mathcal{M})$ we have

$$
\left\|\left(x_{k}\right)_{k \geq 1}\right\|_{H_{c}^{E}+H_{r}^{E}} \lesssim_{E}\left\|\left(y_{k}\right)_{k \geq 1}\right\|_{E\left(\mathcal{M} ; \ell^{\infty}\right)} \lesssim_{E}\left\|\left(x_{k}\right)_{k \geq 1}\right\|_{H_{c}^{E} \cap H_{r}^{E}} .
$$

Suppose that, moreover, $E$ is separable. If $p_{E}>1$ and either $q_{E}<2$ or $E$ is 2-concave, then

$$
\left\|\left(y_{k}\right)_{k \geq 1}\right\|_{E\left(\mathcal{M} ; \ell^{\infty}\right)} \simeq_{E}\left\|\left(x_{k}\right)\right\|_{H_{c}^{E}+H_{r}^{E}} .
$$

On the other hand, if either $E$ is 2 -convex and $q_{E}<\infty$ or $2<p_{E} \leq q_{E}<\infty$, then

$$
\left\|\left(y_{k}\right)_{k \geq 1}\right\|_{E\left(\mathcal{M} ; \ell^{\infty}\right)} \simeq_{E}\left\|\left(x_{k}\right)\right\|_{H_{c}^{E} \cap H_{r}^{E}} .
$$

Proof. If $F$ is a symmetric Banach function space with $F^{\times}=E$, then by (5) $1<p_{F} \leq q_{F}<\infty$. By Theorem 5.7.

$$
\left\|\sum_{k \geq 1} x_{k}\right\|_{E(\mathcal{M})} \simeq_{E}\left\|\left(\sum_{k=1}^{n} x_{k}\right)_{n \geq 1}\right\|_{E\left(\mathcal{M} ; \ell^{\infty}\right)} .
$$

The result now follows directly from [7, Proposition 4.18.

The following result generalizes the noncommutative Rosenthal inequalities presented in [7, as well as the Burkholder-Rosenthal inequalities for noncommutative $L^{p}$-spaces and Lorentz spaces obtained in [18, Theorem 5.1, and [15], Theorem 3.1 , respectively. In fact, Theorem 6.2 positively answers an open question posed in [16], Problem 3.5(2). The proof follows the general strategy of the proof of [7], Theorem 6.3. Let $M_{n}(\mathcal{M})$ denote the von Neumann algebra of $n \times n$ matrices with entries in $\mathcal{M}$ and for any sequence $\left(x_{k}\right)_{k=1}^{n}$ in $E(\mathcal{M})$ let $\operatorname{diag}\left(x_{k}\right)$ and $\operatorname{col}\left(x_{k}\right)$ be the matrices with the $x_{k}$ on the diagonal and first column, respectively, and zeroes elsewhere.

Theorem 6.2 (Noncommutative Burkholder-Rosenthal inequalities). Let $\mathcal{M}$ be a semi-finite von Neumann algebra. Suppose that $E$ is a symmetric Banach function space on $\mathbb{R}_{+}$satisfying $2<p_{E} \leq q_{E}<\infty$. Let $\left(\mathcal{M}_{k}\right)$ be a filtration in $\mathcal{M}$, and for every $k \geq 1$, let $\mathcal{E}_{k}$ denote the conditional expectation with respect to $\mathcal{M}_{k}$. Let $\left(x_{k}\right)$ 
be a martingale difference sequence in $E(\mathcal{M})$ with respect to $\left(\mathcal{M}_{k}\right)$. Then, for any $n \geq 1$,

$$
\begin{gathered}
\left\|\sum_{k=1}^{n} x_{k}\right\|_{E(\mathcal{M})} \simeq_{E} \quad \max \left\{\left\|\operatorname{diag}\left(x_{k}\right)_{k=1}^{n}\right\|_{E\left(M_{n}(\mathcal{M})\right)},\left\|\left(\sum_{k=1}^{n} \mathcal{E}_{k-1}\left|x_{k}\right|^{2}\right)^{\frac{1}{2}}\right\|_{E(\mathcal{M})},\right. \\
\left.\left\|\left(\sum_{k=1}^{n} \mathcal{E}_{k-1}\left|x_{k}^{*}\right|^{2}\right)^{\frac{1}{2}}\right\|_{E(\mathcal{M})}\right\} .
\end{gathered}
$$

Proof. We first prove that the maximum on the right hand side is dominated by $\left\|\sum_{k} x_{k}\right\|_{E(\mathcal{M})}$. Recall that $L^{q}(\mathcal{M})$ has cotype $q$ if $2 \leq q<\infty$, i.e.,

$$
\left\|\operatorname{diag}\left(x_{k}\right)_{k=1}^{n}\right\|_{L^{q}\left(M_{n}(\mathcal{M})\right)}=\left(\sum_{k=1}^{n}\left\|x_{k}\right\|_{L^{q}(\mathcal{M})}^{q}\right)^{\frac{1}{q}} \leq\left\|\sum_{k=1}^{n} r_{k} \otimes x_{k}\right\|_{L^{q}\left(L^{\infty} \bar{\otimes} \mathcal{M}\right)} .
$$

By interpolating this estimate for $q=2$ and $q>q_{E}$ we obtain

$$
\left\|\operatorname{diag}\left(x_{k}\right)_{k=1}^{n}\right\|_{E\left(M_{n}(\mathcal{M})\right)} \lesssim_{E}\left\|\sum_{k=1}^{n} r_{k} \otimes x_{k}\right\|_{E\left(L^{\infty} \bar{\otimes} \mathcal{M}\right)} .
$$

Moreover, by [7], Lemma 4.17,

$$
\left\|\sum_{k} r_{k} \otimes x_{k}\right\|_{E\left(L^{\infty} \bar{\otimes} \mathcal{M}\right)} \simeq_{E}\left\|\sum_{k} x_{k}\right\|_{E(\mathcal{M})}
$$

Since $1<p_{E_{(2)}} \leq q_{E_{(2)}}<\infty$, we obtain by applying the noncommutative dual Doob inequality (Corollary 4.13) in $E_{(2)}(\mathcal{M})$,

$$
\begin{aligned}
\left\|\left(\sum_{k} \mathcal{E}_{k-1}\left(x_{k}^{*} x_{k}\right)\right)^{\frac{1}{2}}\right\|_{E(\mathcal{M})} & =\left\|\sum_{k} \mathcal{E}_{k-1}\left(x_{k}^{*} x_{k}\right)\right\|_{E_{(2)}(\mathcal{M})}^{\frac{1}{2}} \\
& \lesssim_{E}\left\|\sum_{k} x_{k}^{*} x_{k}\right\|_{E_{(2)}(\mathcal{M})}^{\frac{1}{2}}=\left\|\left(\sum_{k} x_{k}^{*} x_{k}\right)^{\frac{1}{2}}\right\|_{E(\mathcal{M})} .
\end{aligned}
$$

Therefore, by the noncommutative Burkholder-Gundy inequality ([7, Proposition 4.18) we conclude that

$$
\left\|\left(\sum_{k} \mathcal{E}_{k-1}\left(x_{k}^{*} x_{k}\right)\right)^{\frac{1}{2}}\right\|_{E(\mathcal{M})} \lesssim_{E}\left\|\left(\sum_{k} x_{k}^{*} x_{k}\right)^{\frac{1}{2}}\right\|_{E(\mathcal{M})} \lesssim_{E}\left\|\sum_{k} x_{k}\right\|_{E(\mathcal{M})},
$$

and by applying this to the sequence $\left(x_{k}^{*}\right)$ we get

$$
\left\|\left(\sum_{k} \mathcal{E}_{k-1}\left(x_{k} x_{k}^{*}\right)\right)^{\frac{1}{2}}\right\|_{E(\mathcal{M})} \lesssim E\left\|\sum_{k} x_{k}\right\|_{E(\mathcal{M})} .
$$

We now prove the reverse inequality in (34). By the noncommutative BurkholderGundy inequality,

$$
\left\|\sum_{k} x_{k}\right\|_{E(\mathcal{M})} \lesssim_{E} \max \left\{\left\|\left(\sum_{k} x_{k}^{*} x_{k}\right)^{\frac{1}{2}}\right\|_{E(\mathcal{M})},\left\|\left(\sum_{k} x_{k} x_{k}^{*}\right)^{\frac{1}{2}}\right\|_{E(\mathcal{M})}\right\} .
$$


By the quasi-triangle inequality in $E_{(2)}(\mathcal{M})$ we have

$$
\begin{aligned}
& \left\|\left(\sum_{k} x_{k}^{*} x_{k}\right)^{\frac{1}{2}}\right\|_{E(\mathcal{M})} \\
& \quad \lesssim_{E}\left(\left\|\sum_{k} x_{k}^{*} x_{k}-\mathcal{E}_{k-1}\left(x_{k}^{*} x_{k}\right)\right\|_{E_{(2)}(\mathcal{M})}+\left\|\sum_{k} \mathcal{E}_{k-1}\left(x_{k}^{*} x_{k}\right)\right\|_{E_{(2)}(\mathcal{M})}\right)^{\frac{1}{2}} .
\end{aligned}
$$

Notice that $\left(\left|x_{k}\right|^{2}-\mathcal{E}_{k-1}\left(\left|x_{k}\right|^{2}\right)\right)_{k \geq 1}$ is a martingale difference sequence in $E_{(2)}(\mathcal{M})$. Since $1<p_{E_{(2)}} \leq q_{E_{(2)}}<\infty$ we find by the noncommutative Burkholder-Gundy inequality

$$
\begin{array}{r}
\left\|\sum_{k} x_{k}^{*} x_{k}-\mathcal{E}_{k-1}\left(x_{k}^{*} x_{k}\right)\right\|_{E_{(2)}(\mathcal{M})} \lesssim_{E}\left\|\left(\sum_{k}\left(x_{k}^{*} x_{k}-\mathcal{E}_{k-1}\left(x_{k}^{*} x_{k}\right)\right)^{2}\right)^{\frac{1}{2}}\right\|_{E_{(2)}(\mathcal{M})} \\
\lesssim_{E}\left\|\left(\sum_{k}\left|x_{k}\right|^{4}\right)^{\frac{1}{2}}\right\|_{E_{(2)}(\mathcal{M})}+\left\|\left(\sum_{k}\left(\mathcal{E}_{k-1}\left|x_{k}\right|^{2}\right)^{2}\right)^{\frac{1}{2}}\right\|_{E_{(2)}(\mathcal{M})},
\end{array}
$$

where in the final inequality we use the quasi-triangle inequality in $E_{(2)}\left(\mathcal{M} ; \ell_{c}^{2}\right)$. By applying the noncommutative Stein inequality (Corollary 4.16) to the second term on the right hand side, we find that

$$
\left\|\sum_{k} x_{k}^{*} x_{k}-\mathcal{E}_{k-1}\left(x_{k}^{*} x_{k}\right)\right\|_{E_{(2)}(\mathcal{M})} \lesssim_{E}\left\|\left(\sum_{k}\left|x_{k}\right|^{4}\right)^{\frac{1}{2}}\right\|_{E_{(2)}(\mathcal{M})} .
$$

Let $x=\operatorname{col}\left(\left|x_{k}\right|\right)$ and $y=\operatorname{diag}\left(\left|x_{k}\right|\right)$. Since $\mu(y x) \prec \prec \mu(x) \mu(y)$, it follows from the Calderón-Mitjagin theorem ([23], Theorem II.3.4) that there is a contraction $T$ for the couple $\left(L^{1}, L^{\infty}\right)$ such that $\mu(y x)=T(\mu(x) \mu(y))$. Therefore,

$$
\begin{aligned}
\left\|\left(\sum_{k}\left|x_{k}\right|^{4}\right)^{\frac{1}{2}}\right\|_{E_{(2)}(\mathcal{M})} & =\left\|\left(x^{*} y^{*} y x\right)^{\frac{1}{2}}\right\|_{E_{(2)}\left(M_{n}(\mathcal{M})\right)} \\
& =\|y x\|_{E_{(2)}\left(M_{n}(\mathcal{M})\right) \lesssim E}\|\mu(x) \mu(y)\|_{E_{(2)}} \\
& =\left\|\mu(x)^{\frac{1}{2}} \mu(y)^{\frac{1}{2}}\right\|_{E}^{2} \leq\|y\|_{E\left(M_{n}(\mathcal{M})\right)}\|x\|_{E\left(M_{n}(\mathcal{M})\right)} \\
& =\left\|\operatorname{diag}\left(x_{k}\right)\right\|_{E\left(M_{n}(\mathcal{M})\right)}\left\|\left(\sum_{k}\left|x_{k}\right|^{2}\right)^{\frac{1}{2}}\right\|_{E(\mathcal{M})},
\end{aligned}
$$

where in the final inequality we use Hölder's inequality. Putting our estimates together, starting from (36), we arrive at

$$
\begin{aligned}
& \left\|\left(\sum_{k}\left|x_{k}\right|^{2}\right)^{\frac{1}{2}}\right\|_{E(\mathcal{M})} \\
& \quad \lesssim_{E}\left(\left\|\operatorname{diag}\left(x_{k}\right)\right\|_{E\left(M_{n}(\mathcal{M})\right)}\left\|\left(\sum_{k}\left|x_{k}\right|^{2}\right)^{\frac{1}{2}}\right\|_{E(\mathcal{M})}+\left\|\left(\sum_{k} \mathcal{E}_{k-1}\left|x_{k}\right|^{2}\right)^{\frac{1}{2}}\right\|_{E(\mathcal{M})}^{2}\right)^{\frac{1}{2}} .
\end{aligned}
$$

In other words, if we set $a=\left\|\left(\sum_{k}\left|x_{k}\right|^{2}\right)^{\frac{1}{2}}\right\|_{E(\mathcal{M})}, b=\left\|\operatorname{diag}\left(x_{k}\right)\right\|_{E\left(M_{n}(\mathcal{M})\right)}$ and $c=\left\|\left(\sum_{k} \mathcal{E}_{k-1}\left|x_{k}\right|^{2}\right)^{\frac{1}{2}}\right\|_{E(\mathcal{M})}$, we have $a^{2} \lesssim_{E} a b+c^{2}$. Solving this quadratic equation we obtain $a \lesssim_{E} \max \{b, c\}$ or

$$
\left\|\left(\sum_{k}\left|x_{k}\right|^{2}\right)^{\frac{1}{2}}\right\|_{E(\mathcal{M})} \lesssim_{E} \max \left\{\left\|\operatorname{diag}\left(x_{k}\right)\right\|_{E\left(M_{n}(\mathcal{M})\right)},\left\|\left(\sum_{k} \mathcal{E}_{k-1}\left|x_{k}\right|^{2}\right)^{\frac{1}{2}}\right\|_{E(\mathcal{M})}\right\} .
$$


Applying this to the sequence $\left(x_{k}^{*}\right)$ gives

$$
\left\|\left(\sum_{k}\left|x_{k}^{*}\right|^{2}\right)^{\frac{1}{2}}\right\|_{E(\mathcal{M})} \lesssim_{E} \max \left\{\left\|\operatorname{diag}\left(x_{k}\right)\right\|_{E\left(M_{n}(\mathcal{M})\right)},\left\|\left(\sum_{k} \mathcal{E}_{k-1}\left|x_{k}^{*}\right|^{2}\right)^{\frac{1}{2}}\right\|_{E(\mathcal{M})}\right\} .
$$

The result now follows by (35).

Remark 6.3. Of course, if $E$ is the Köthe dual of a separable symmetric Banach function space on $\mathbb{R}_{+}$, then by Theorem 5.7 we may replace $\left\|\sum_{k=1}^{n} x_{k}\right\|_{E(\mathcal{M})}$ by $\left\|\left(y_{k}\right)_{k=1}^{n}\right\|_{E(\mathcal{M} ; \ell)}$ in (34), where $y_{k}=\sum_{i=1}^{k} x_{i}$.

\section{Appendix A. Original approach to Boyd's theOREM}

In this appendix we prove a full noncommutative analogue of Boyd's interpolation theorem, which allows for interpolation of operators of weak type instead of only operators of Marcinkiewicz weak type. We adapt the original proof of Boyd [5].

The first result is an extension of Calderón's characterization of weak type operators ([6], Theorem 8). For $0<p<q<\infty$ define Calderón's operator by

$$
S_{p, q} f(t)=t^{-\frac{1}{p}} \int_{0}^{t} s^{\frac{1}{p}} f(s) \frac{d s}{s}+t^{-\frac{1}{q}} \int_{t}^{\infty} s^{\frac{1}{q}} f(s) \frac{d s}{s} \quad\left(t>0, f \in S\left(\mathbb{R}_{+}\right)\right)
$$

and set

$$
S_{p, \infty} f(t)=t^{-\frac{1}{p}} \int_{0}^{t} s^{\frac{1}{p}} f(s) \frac{d s}{s} \quad\left(t>0, f \in S\left(\mathbb{R}_{+}\right)\right) .
$$

Theorem A.1. Let $\mathcal{M}, \mathcal{N}$ be von Neumann algebras equipped with normal, semifinite, faithful traces $\tau$ and $\sigma$, respectively. Let $0<p<q \leq \infty$ and suppose that $T: L^{p, 1}(\mathcal{M})_{+}+L^{q, 1}(\mathcal{M})_{+} \rightarrow S(\sigma)_{+}$is a midpoint convex map satisfying

$$
\|T x\|_{L^{r, \infty}(\mathcal{N})} \leq C_{r}\|x\|_{L^{r, 1}(\mathcal{M})} \quad\left(x \in L^{r, 1}(\mathcal{M})_{+}, r=p, q\right) .
$$

Then,

$$
\mu_{t}(T x) \lesssim_{p, q} \max \left\{C_{p}, C_{q}\right\} S_{p, q} \mu(x)(t) \quad(t>0) .
$$

Proof. Suppose that $q<\infty$. We may assume that $\max \left\{C_{p}, C_{q}\right\} \leq 1$. Fix $t>0$, let $x \in L^{p, 1}(\mathcal{M})_{+}+L^{q, 1}(\mathcal{M})_{+}$and set $\delta=\mu_{t}(x)$. Define $x_{t}^{1}=(x-\delta) e^{x}(\delta, \infty)$ and $x_{t}^{2}=x-x_{t}^{1}$, so $x_{t}^{2}=x e^{x}[0, \delta]+\delta e^{x}(\delta, \infty)$. Observe that $x_{t}^{1}, x_{t}^{2} \geq 0$ as $x \geq 0$. Define two increasing, continuous functions $\phi_{1}, \phi_{2}: \mathbb{R}_{+} \rightarrow \mathbb{R}_{+}$by

$$
\phi_{1}(u)=(u-\delta) \chi_{\{u>\delta\}}, \quad \phi_{2}(u)=\delta \chi_{\{u>\delta\}}+u \chi_{\{u \leq \delta\}} .
$$

From Proposition 4.1 it follows that

$$
\begin{aligned}
& \mu\left(x_{t}^{1}\right)=\mu\left(\phi_{1}(x)\right)=\phi_{1}(\mu(x))=(\mu(x)-\delta) \chi_{\{\mu(x) \geq \delta\}} \\
& \mu\left(x_{t}^{2}\right)=\mu\left(\phi_{2}(x)\right)=\phi_{2}(\mu(x))=\delta \chi_{\{\mu(x)>\delta\}}+\mu(x) \chi_{\{\mu(x) \leq \delta\}} .
\end{aligned}
$$

Using that $\delta=\mu_{t}(x)$ we obtain

$$
\begin{aligned}
& \mu_{s}\left(x_{t}^{1}\right)=\left(\mu_{s}(x)-\delta\right) \chi_{[0, t]}(s) \quad(s>0) ; \\
& \mu_{s}\left(x_{t}^{2}\right)=\delta \chi_{[0, t]}(s)+\mu_{s}(x) \chi_{(t, \infty)}(s) \quad(s>0) .
\end{aligned}
$$

In particular, $\mu(x)=\mu\left(x_{t}^{1}\right)+\mu\left(x_{t}^{2}\right)$. By midpoint convexity,

$$
\begin{aligned}
\mu_{t}(T x) & \leq \mu_{t}\left(\frac{1}{2} T\left(2 x_{t}^{1}\right)+\frac{1}{2} T\left(2 x_{t}^{2}\right)\right) \\
& \leq \frac{1}{2} \mu_{\frac{t}{2}}\left(T\left(2 x_{t}^{1}\right)\right)+\frac{1}{2} \mu_{\frac{t}{2}}\left(T\left(2 x_{t}^{2}\right)\right) .
\end{aligned}
$$


By (38) we obtain

$$
\mu_{\frac{t}{2}}\left(T\left(2 x_{t}^{1}\right)\right) \lesssim_{p} t^{-\frac{1}{p}}\left\|x_{t}^{1}\right\|_{L^{p, 1}(\mathcal{M})}=t^{-\frac{1}{p}} \int_{0}^{t} s^{\frac{1}{p}} \mu_{s}\left(x_{t}^{1}\right) \frac{d s}{s}=S_{p, q} \mu\left(x_{t}^{1}\right)(t)
$$

and, moreover,

$$
\begin{aligned}
\mu_{\frac{t}{2}}\left(T\left(2 x_{t}^{2}\right)\right) & \lesssim q t^{-\frac{1}{q}}\left\|x_{t}^{2}\right\|_{L^{q, 1}(\mathcal{M})} \\
& =t^{-\frac{1}{q}} \int_{0}^{\infty} s^{\frac{1}{q}} \mu_{s}\left(x_{t}^{2}\right) \frac{d s}{s} \\
& =t^{-\frac{1}{q}} \int_{0}^{t} s^{\frac{1}{q}} \delta \frac{d s}{s}+t^{-\frac{1}{q}} \int_{t}^{\infty} s^{\frac{1}{q}} \mu_{s}(x) \frac{d s}{s} .
\end{aligned}
$$

Since

$$
t^{-\frac{1}{q}} \int_{0}^{t} s^{\frac{1}{q}} \delta \frac{d s}{s}=q \delta=\frac{q}{p} t^{-\frac{1}{p}} \int_{0}^{t} s^{\frac{1}{p}} \delta \frac{d s}{s}
$$

it follows that

$$
\mu_{\frac{t}{2}}\left(T\left(2 x_{t}^{2}\right)\right) \lesssim_{p, q} S_{p, q} \mu\left(x_{t}^{2}\right)(t) .
$$

Putting our estimates together, we conclude that

$$
\mu_{t}(T x) \lesssim_{p, q} S_{p, q} \mu\left(x_{t}^{1}\right)(t)+S_{p, q} \mu\left(x_{t}^{2}\right)(t)=S_{p, q} \mu(x)(t) .
$$

The case $q=\infty$ is proved similarly.

The following observation for symmetric Banach function spaces and $p, q \geq 1$ is the main result of [5]. The general case is proved using essentially the same argument (see 29], Theorem 2).

Theorem A.2. If $E$ is a symmetric quasi-Banach function space on $\mathbb{R}_{+}$, then the following hold.

(a) If $0<p<q<\infty$, then $S_{p, q}$ is bounded on $E$ if and only if $p<p_{E} \leq q_{E}<q$.

(b) If $0<p<\infty$, then $S_{p, \infty}$ is bounded on $E$ if and only if $p<p_{E}$.

By combining the observations in Theorems A.1 and A.2 we find the following noncommutative extension of Boyd's theorem.

Theorem A.3. Let $E$ be a symmetric quasi-Banach function space on $\mathbb{R}_{+}$which is s-convex for some $0<s<\infty$. Let $\mathcal{M}, \mathcal{N}$ be von Neumann algebras equipped with normal, semi-finite, faithful traces $\tau$ and $\sigma$, respectively. Suppose that $0<p<q \leq$ $\infty$ and let $T: L^{p, 1}(\mathcal{M})_{+}+L^{q, 1}(\mathcal{M})_{+} \rightarrow S(\sigma)_{+}$be a midpoint convex map such that

$$
\|T x\|_{L^{r, \infty}(\mathcal{N})} \leq C_{r}\|x\|_{L^{r, 1}(\mathcal{M})} \quad\left(x \in L^{r, 1}(\mathcal{M})_{+}, r=p, q\right) .
$$

If $p<p_{E} \leq q_{E}<q<\infty$ or $p<p_{E}$ and $q=\infty$, then

$$
\|T x\|_{E(\mathcal{N})} \lesssim_{p, q} \max \left\{C_{p}, C_{q}\right\}\left\|S_{p, q}\right\|_{E \rightarrow E}\|x\|_{E(\mathcal{M})} \quad\left(x \in E(\mathcal{M})_{+}\right) .
$$

\section{ACKNOWLEDGEMENT}

The author would like to thank the anonymous reviewer for comments on an earlier version of this paper and the resulting improvements. 


\section{REFERENCES}

[1] Turdebek N. Bekjan and Zeqian Chen, Interpolation and $\Phi$-moment inequalities of noncommutative martingales, Probab. Theory Related Fields 152 (2012), no. 1-2, 179-206, DOI 10.1007/s00440-010-0319-2. MR2875756

[2] T. Bekjan, Z. Chen, and A. Osȩkowski, Noncommutative moment maximal inequalities. arXiv: 1108.2795

[3] A. Benedek, A.-P. Calderón, and R. Panzone, Convolution operators on Banach space valued functions, Proc. Nat. Acad. Sci. U.S.A. 48 (1962), 356-365. MR0133653 (24 \#A3479)

[4] Colin Bennett and Robert Sharpley, Interpolation of operators, Pure and Applied Mathematics, vol. 129, Academic Press, Inc., Boston, MA, 1988. MR928802 (89e:46001)

[5] David W. Boyd, Indices of function spaces and their relationship to interpolation, Canad. J. Math. 21 (1969), 1245-1254. MR0412788 (54 \#909)

[6] A.-P. Calderón, Spaces between $L^{1}$ and $L^{\infty}$ and the theorem of Marcinkiewicz, Studia Math. 26 (1966), 273-299. MR0203444 (34 \#3295)

[7] Sjoerd Dirksen, Ben de Pagter, Denis Potapov, and Fedor Sukochev, Rosenthal inequalities in noncommutative symmetric spaces, J. Funct. Anal. 261 (2011), no. 10, 2890-2925, DOI 10.1016/j.jfa.2011.07.015. MR2832586 (2012k:46073)

[8] S. Dirksen and É. Ricard, Some remarks on noncommutative Khintchine inequalities, Bull. London Math. Soc. 45 (2013), no. 3, 618-624. MR3065031

[9] Peter G. Dodds, Theresa K.-Y. Dodds, and Ben de Pagter, Noncommutative Banach function spaces, Math. Z. 201 (1989), no. 4, 583-597, DOI 10.1007/BF01215160. MR.1004176 (90j:46054)

[10] Peter G. Dodds, Theresa K. Dodds, and Ben de Pagter, Fully symmetric operator spaces, Integral Equations Operator Theory 15 (1992), no. 6, 942-972, DOI 10.1007/BF01203122. MR.1188788 (94j:46062)

[11] Peter G. Dodds, Theresa K.-Y. Dodds, and Ben de Pagter, Noncommutative Köthe duality, Trans. Amer. Math. Soc. 339 (1993), no. 2, 717-750, DOI 10.2307/2154295. MR.1113694 (94a:46093)

[12] P. Dodds, B. de Pagter, and F. Sukochev, Noncommutative integration. Work in progress.

[13] Thierry Fack and Hideki Kosaki, Generalized s-numbers of $\tau$-measurable operators, Pacific J. Math. 123 (1986), no. 2, 269-300. MR.840845 (87h:46122)

[14] Ying Hu, Noncommutative extrapolation theorems and applications, Illinois J. Math. 53 (2009), no. 2, 463-482. MR2594639 (2011c:46137)

[15] Yong Jiao, Burkholder's inequalities in noncommutative Lorentz spaces, Proc. Amer. Math. Soc. 138 (2010), no. 7, 2431-2441, DOI 10.1090/S0002-9939-10-10267-6. MR2607873 (2011f:46080)

[16] Yong Jiao, Martingale inequalities in noncommutative symmetric spaces, Arch. Math. (Basel) 98 (2012), no. 1, 87-97, DOI 10.1007/s00013-011-0343-1. MR2885535

[17] Marius Junge, Doob's inequality for non-commutative martingales, J. Reine Angew. Math. 549 (2002), 149-190, DOI 10.1515/crll.2002.061. MR.1916654 (2003k:46097)

[18] Marius Junge and Quanhua Xu, Noncommutative Burkholder/Rosenthal inequalities, Ann. Probab. 31 (2003), no. 2, 948-995, DOI 10.1214/aop/1048516542. MR.1964955 (2004f:46078)

[19] Marius Junge and Quanhua Xu, Noncommutative maximal ergodic theorems, J. Amer. Math. Soc. 20 (2007), no. 2, 385-439, DOI 10.1090/S0894-0347-06-00533-9. MR2276775 (2007k:46109)

[20] N. J. Kalton, Compact and strictly singular operators on certain function spaces, Arch. Math. (Basel) 43 (1984), no. 1, 66-78, DOI 10.1007/BF01193613. MR758342 (85m:47027)

[21] N. J. Kalton, N. T. Peck, and James W. Roberts, An F-space sampler, London Mathematical Society Lecture Note Series, vol. 89, Cambridge University Press, Cambridge, 1984. MR.808777 (87c:46002)

[22] N. J. Kalton and F. A. Sukochev, Symmetric norms and spaces of operators, J. Reine Angew. Math. 621 (2008), 81-121, DOI 10.1515/CRELLE.2008.059. MR2431251 (2009i:46118)

[23] S. Krĕ̌n, Y. Petunīn, and E. Semënov, Interpolation of linear operators, American Mathematical Society, Providence, R.I., 1982. MR0649411 (84j:46103)

[24] Joram Lindenstrauss and Lior Tzafriri, Classical Banach spaces. II, Function spaces. Ergebnisse der Mathematik und ihrer Grenzgebiete [Results in Mathematics and Related Areas], vol. 97, Springer-Verlag, Berlin-New York, 1979. MR.540367 (81c:46001) 
[25] Françoise Lust-Piquard, Inégalités de Khintchine dans $C_{p}(1<p<\infty)$ (French, with English summary), C. R. Acad. Sci. Paris Sér. I Math. 303 (1986), no. 7, 289-292. MR859804 $(87 \mathrm{j}: 47032)$

[26] Françoise Lust-Piquard and Gilles Pisier, Noncommutative Khintchine and Paley inequalities, Ark. Mat. 29 (1991), no. 2, 241-260, DOI 10.1007/BF02384340. MR.1150376 (94b:46011)

[27] Lech Maligranda, Indices and interpolation, Dissertationes Math. (Rozprawy Mat.) 234 (1985), 49. MR820076 (87k:46059)

[28] J. Marcinkiewicz, Sur l'interpolation d'opérations, C. R. Acad. Sci., Paris, 208:1272-1273, 1939.

[29] Stephen J. Montgomery-Smith, The Hardy operator and Boyd indices, Interaction between functional analysis, harmonic analysis, and probability (Columbia, MO, 1994), Lecture Notes in Pure and Appl. Math., vol. 175, Dekker, New York, 1996, pp. 359-364. MR1358172 (97a:46037)

[30] Edward Nelson, Notes on non-commutative integration, J. Functional Analysis 15 (1974), 103-116. MR0355628 (50 \#8102)

[31] Gilles Pisier, Non-commutative vector valued $L_{p}$-spaces and completely p-summing maps (English, with English and French summaries), Astérisque 247 (1998), vi+131. MR1648908 (2000a:46108)

[32] Gilles Pisier and Quanhua Xu, Non-commutative martingale inequalities, Comm. Math. Phys. 189 (1997), no. 3, 667-698, DOI 10.1007/s002200050224. MR1482934 (98m:46079)

[33] E. M. Stein and Guido Weiss, An extension of a theorem of Marcinkiewicz and some of its applications, J. Math. Mech. 8 (1959), 263-284. MR0107163 (21 \#5888)

[34] Q. Xu, Operator spaces and noncommutative $L_{p}$, Nankai University summer school lecture notes, 2007.

[35] Quan Hua Xu, Analytic functions with values in lattices and symmetric spaces of measurable operators, Math. Proc. Cambridge Philos. Soc. 109 (1991), no. 3, 541-563, DOI 10.1017/S030500410006998X. MR1094753 (92g:46036)

[36] A. Zygmund, On a theorem of Marcinkiewicz concerning interpolation of operations, J. Math. Pures Appl. (9) 35 (1956), 223-248. MR0080887 (18,321d)

Hausdorff Center for Mathematics, Universität Bonn, Endenicher Allee 60, 53115 Bonn, Germany

E-mail address: sjoerd.dirksen@hcm.uni-bonn.de

Current address: Department of Mathematics, RWTH Aachen University, 52056 Aachen, Germany

E-mail address: dirksen@mathc.rwth-aachen.de 